\title{
The dynamics around the collinear equilibrium points of the RTBP
}

\author{
G. GÓMEZ1 ${ }^{1}$ and J.M. MONDELO² (corresponding author) \\ ${ }^{1}$ Departament de Matemàtica Aplicada i Anàlisi, Universitat de Barcelona \\ Gran Via 585, 08007 Barcelona, Spain, email: gomez@cerber.mat.ub.es \\ ${ }^{2}$ Dept. de Matemàtica Aplicada I, Universitat Politècnica de Catalunya \\ Diagonal 647, 08028 Barcelona, Spain, email: mondelo@ma1.upc.es \\ phone: (34) 9340160 67, fax: (34) 934011713
}

\begin{abstract}
This paper is devoted to the analysis of an extended neighborhood of the collinear equilibrium points of the Restricted Three Body Problem. The analysis is done using numerical tools for the determination of periodic orbits and invariant 2D tori. All the relevant information of the neutrally stable behavior of the dynamics in the vicinity of the three libration points is given. The results in this paper extend those given in [11], since they avoid the convergence restrictions of the semi-analytical approach used in this reference.
\end{abstract}

PACS codes: 95.10.Ce, 02.60.Cb, 02.40.Vh

Keywords: Invariant tori, Halo orbits, Lissajous orbits, RTBP, parallel computing.

\section{Introduction}

Much attention has been paid in the past to the study of motion in the vicinity of the collinear equilibrium points of the Restricted Three Body Problem (RTBP). This is because part of the dynamics of the RTBP is "organized" by the equilibrium points and their invariant unstable and stable manifolds (see [14],[13]). Another reason, useful for applications, is that near these points there are orbits which have nice properties for spacecraft missions, since they can be used to set permanent observatories of the Sun, the magnetosphere of the Earth, links with the hidden part of the Moon, and others.

The goal of this paper is to perform a systematic and, as far as possible, complete study of the dynamics in a large neighborhood of the three collinear equilibrium points for different energy levels. Because of its interest for spacecraft mission design, all the study has been done for the value of the mass parameter $\mu=0.012150585$, corresponding to the Earth-Moon system. In a forthcoming paper we will show how the dynamics around these equilibrium points evolves with $\mu$ as well as how it varies when more perturbing bodies are included in the model. 
The collinear libration points behave, linearly, as the product of two centers by a saddle. Hence, one can expect families of periodic orbits which in the limit have frequencies related to both centers: $\omega_{p}$ and $\omega_{v}$ (called planar and vertical frequencies, respectively). This is assured by the Lyapunov center theorem, unless one of the frequencies is an integer multiple of the other (which only happens for a countable set of values of the mass ratio (see [16])). Near the libration points we can also expect $2 \mathrm{D}$ tori, with basic frequencies that when we approach to the point go to $\omega_{p}$ and $\omega_{v}$. The rigorous existence of these tori is more problematic. First, the basic frequencies at the collinear point can be too close to resonant. Furthermore, the frequencies change with their amplitudes and so, they go across resonances when the amplitudes are changed. This leads to a Cantor set of tori. The proof of the existence of these tori follows similar lines to the proof of the KAM theorem (see [12]).

Due to the saddle component of the linear approximation, close to the $L_{1}$ and $L_{2}$ libration points the dynamics is that of a strong unstable equilibrium. However, due to the center $\times$ center part and when we consider all the energy levels, there are 4D center manifolds around them (they are also called neutrally stable manifolds). On a given energy level this is just a 3D set where the dynamics has a "neutral behavior". On it there are periodic orbits and 2D invariant tori. The $L_{3}$ point has the same linear behavior, however the instability is quite mild. A body close to the $L_{3}$ point in the Earth-Sun system (assuming no more bodies in the solar system) would remain close to it for centuries. Nevertheless, the long term effects associated to the unstable/stable manifolds of $L_{3}$ or to the ones of the central manifold around $L_{3}$ are extremely important (see [6], [7]).

For values of the energy close to the one of the equilibrium point, the analysis of the dynamics in the center manifold can be done in a semi-analytical way with different strategies. One consists in performing a reduction of the Hamiltonian that decreases the number of degrees of freedom, removes the hyperbolic directions and allows the numerical study of the Poincaré map in a vicinity of the equilibrium points (see [5] and [11]). This approach is usually known as the reduction to the center manifold. Note that, generically, the expansions required for these computations cannot be convergent in any open set, because of the crossing of resonances. Another procedure consists in the use of Lindstedt-Poincaré methods to explicitly compute the periodic orbits and invariant tori (see [5], [7], [9], [15]). It looks for an analytical expressions for them in terms of suitable amplitudes and phases. Both approaches are limited by the convergence of the expansions used, for the changes of coordinates and the Hamiltonian in the first case and for the periodic orbits and invariant tori in the second, which is discussed in the above mentioned papers.

In this study we compute the periodic orbits and invariant 2D tori of the center manifolds of the three collinear libration points using purely numerical procedures. With this approach, we can extend the analysis of the phase space done with semi-analytical methods in [5] and [11] to a wider range of energy values, that now include several bifurcations, and also to the $L_{3}$ libration point. Numerical methods have been widely used in the past to compute fixed points and periodic orbits, but there are not many papers dealing with the numerical computation of invariant tori. Following the ideas of [3], we have developed and used a procedure for the refinement and continuation of invariant tori, which is based in the computation of the Fourier series of an invariant curve on the torus. This strategy is combined with a multiple shooting procedure.

In section 2 we will discuss the methodology used, as well as its implementation in a parallel computer. Section 3 is devoted to show the numerical results. For $L_{1}$ and $L_{2}$ some new families of periodic orbits of the halo type will be given that play an important role in the organization of the phase space. These new halo orbits are not in the usual 1:1 resonance, between the in-plane 
and out-off-plane frequencies, but in a 1:p ratio. We will show the results corresponding to $\mathrm{p}=2$ and $\mathrm{p}=3$. To get a global view of the results obtained at each energy level, we will display the full set of orbits computed on the surface of section $\left\{z=0, p_{z}>0\right\}$. In this way it also becomes clear up to what point our results complete those in [5] and [11]. Fig. 1 shows a sample of these results, that are discussed in detail in section 4. This figure displays a "Poincaré map" representation for the libration point trajectories around $L_{1}$. The different curves of the figure represent the intersections with the $(x, y)$ plane, $p_{z}>0$, of the different libration orbits, all of them with the same energy value. Clearly, the figure is symmetric with respect to $y=0$, which reflects one of the natural symmetries of the RTBP. The most outer curves in the figure are the intersections with $z=0$ of $2 \mathrm{D}$ tori around the planar Lyapunov orbit around $L_{1}$. On the $y=0$ axis there are three fixed points: those close to $x \approx-0.92$ and $x \approx-0.79$ correspond to the intersections of two periodic orbits, each one in a different lane of a bridge of periodic orbits that connects the planar and the vertical Lyapunov families (see section 3.1). The fixed point close to $x \approx-0.86$ represents the vertical Lyapunov periodic orbit. Around this last point there appear some elongated curves which are related to tori around the vertical periodic orbits (see section 3.3.1). These tori are usually known as Lissajous trajectories, in the literature of libration point orbits. In the upper and lower parts of the figure there are some additional fixed points, they correspond to the well known halo orbits and to some additional periodic orbits that bifurcate from the families of halo orbits by duplication and triplication of the period (see section 3.2). All of them are surrounded by tori (invariant curves in our representation) since in the normal behaviour of the corresponding periodic orbit an elliptic component is found (see section 3.3.2).

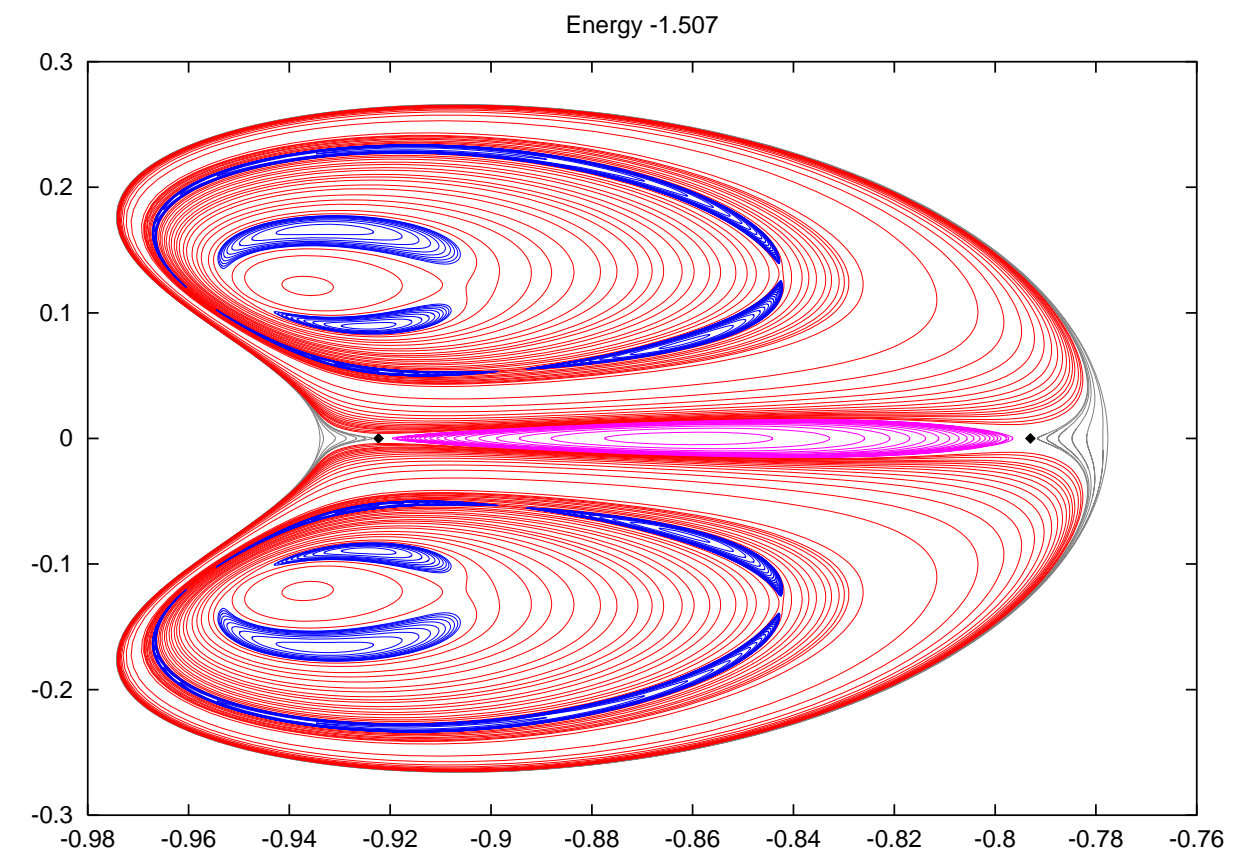

Figure 1: Section with $\left\{z=0, \quad p_{z}>0\right\}$ of the libration point orbits around $L_{1}$ whith energy $h=-1.507$. 


\subsection{Basic equations and notation}

As it is well known, the RTBP describes the motion of a massless particle under the attraction of two bodies of masses $m_{1}$ and $m_{2}$, called primaries, which are assumed to move in circular orbits around their center of mass. Taking a coordinate system that rotates with the primaries and suitable units, the primaries can be assumed to have masses $1-\mu$ and $\mu$ with $\mu \in[0,1 / 2]$, to be fixed at coordinates $(\mu, 0,0)$ and $(\mu-1,0,0)$, and to complete one inertial revolution in $2 \pi$ time units. Under these assumptions, the massless particle is governed by the following second-order differential equations (see [19])

$$
\begin{aligned}
\ddot{x}-2 \dot{y} & =\Omega_{x}, \\
\ddot{y}+2 \dot{x} & =\Omega_{y}, \\
\ddot{z} & =\Omega_{z},
\end{aligned}
$$

where

$$
\Omega=\frac{1}{2}\left(x^{2}+y^{2}\right)+\frac{1-\mu}{r_{1}}+\frac{\mu}{r_{2}}+\frac{1}{2} \mu(1-\mu),
$$

and $r_{1}=\sqrt{(x-\mu)^{2}+y^{2}+z^{2}}, r_{2}=\sqrt{(x-\mu+1)^{2}+y^{2}+z^{2}}$ are the distances from the particles to the primaries. The above system of differential equations has a first integral, called the Jacobi integral, which is defined as

$$
C\left(x, y, z, p_{x}, p_{y}, p_{z}\right)=2 \Omega-\dot{x}^{2}-\dot{y}^{2}-\dot{z}^{2} .
$$

The RTBP has five libration points, two of them, $L_{4}$ and $L_{5}$, form an equilateral triangle with the primaries and are located at $(-1 / 2+\mu, \mp \sqrt{3} / 2,0)$, respectively. The other three are collinear, with $y=z=0$. If $x_{L_{j}}$ denotes the value of the $x$ coordinates for $j=1,2,3$, we will assume that the positions of these points and the primaries are such that

$$
x_{L_{2}}<\mu-1<x_{L_{1}}<\mu<x_{L_{3}} .
$$

For small values of $\mu$, both $\mu-1-x_{L_{2}}$ and $\mu-1-x_{L_{1}}$ are $3^{-1 / 3} \mu^{1 / 3}+O\left(\mu^{2 / 3}\right)$ and $x_{L_{3}}=1+O(\mu)$.

By introducing momenta as $p_{x}=\dot{x}-y, p_{y}=\dot{y}+x$ and $p_{z}=\dot{z}$, the RTBP can be written in Hamiltonian form with Hamiltonian function

$$
H\left(x, y, z, p_{z}, p_{y}, p_{z}\right)=\frac{1}{2}\left(p_{x}^{2}+p_{y}^{2}+p_{z}^{2}\right)-x p_{y}+y p_{x}-\frac{1-\mu}{r_{1}}-\frac{\mu}{r_{2}} .
$$

The differential equations are then

$$
\begin{array}{lll}
\dot{x}=p_{x}+y, & \dot{p}_{x}=p_{y}-\frac{1-\mu}{r_{1}^{3}}(x-\mu)-\frac{\mu}{r_{2}^{3}}(x-\mu+1), \\
\dot{y}=p_{y}-x, & \dot{p}_{y}=-p_{x}-\frac{1-\mu}{r_{1}^{3}} y-\frac{\mu}{r_{2}^{3}} y, \\
\dot{z}=p_{z}, & \dot{p}_{z}=-\frac{1-\mu}{r_{1}^{3}} z-\frac{\mu}{r_{2}^{3}} z .
\end{array}
$$

All the computations that follow have been done using this last set of equations. We will refer to the value of the Hamiltonian as the "energy", and it is related to the Jacobi constant, $C$, by

$$
C=-2 H-\mu(1-\mu) \text {. }
$$


The linearized equations around any collinear equilibrium point are given by the second order terms of the Hamiltonian, which can be written as

$$
H_{2}=\frac{1}{2}\left(p_{x}^{2}+p_{y}^{2}\right)-x p_{y}+y p_{x}-c_{2}\left(x^{2}-\frac{y^{2}}{2}\right)+\frac{p_{z}^{2}}{2}+c_{2} \frac{z^{2}}{2}
$$

where $c_{2}$ is a positive constant (in fact, $c_{2}>1$ ) that depends on the equilibrium point and the value of the mass parameter $\mu$. Its values are represented in Fig. 2 ([15]).

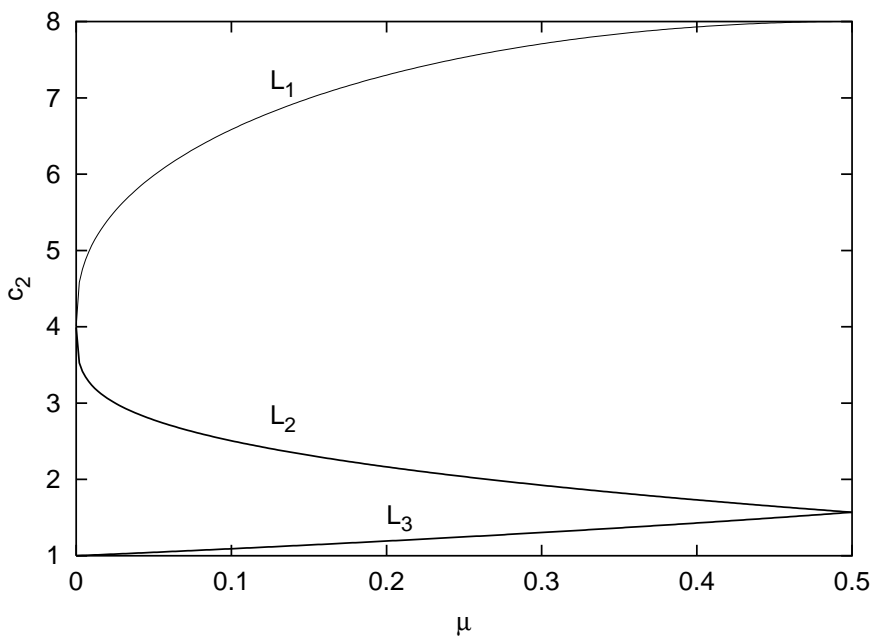

Figure 2: Values of the $c_{2}(\mu)$ function, $\mu \in[0,0.5]$, for $L_{1,2,3}$.

From the above expression for $\mathrm{H}_{2}$, it is clear that, linearly, the $z$ direction is uncoupled form the planar ones and the motion in the vertical direction is an harmonic oscillator with vertical frequency $\omega_{v}=\sqrt{c_{2}}$. The characteristic polynomial associated to the planar motion is

$$
p(\lambda)=\lambda^{4}+\left(2-c_{2}\right) \lambda^{2}+\left(1+c_{2}-2 c_{2}^{2}\right)
$$

Denoting $\eta=\lambda^{2}$, we have that the roots of $p(\lambda)=0$ are given by

$$
\eta_{1,2}=\frac{c_{2}-2 \pm \sqrt{9 c_{2}^{2}-8 c_{2}}}{2}
$$

so, according to the values of $c_{2}$, we have that $\eta_{1}>0$ and $\eta_{2}<0$. This shows that the three equilibrium points are of the type center $\times$ center $\times$ saddle. The constant $\omega_{p}=\sqrt{-\eta_{2}}$ is usually called the planar frequency.

\subsection{Normal behavior around a periodic orbit}

Let $\phi_{t}(\mathbf{x})$ be the flow associated to the Hamiltonian $H(\mathbf{x})$ of the RTBP. The normal behavior of a $T$-periodic orbit through $\mathbf{x}_{0}$ is studied in terms of the time- $T$ flow around $\mathbf{x}_{0}$, whose linear approximation is given by the monodromy matrix $M=D \phi_{T}\left(\mathbf{x}_{0}\right)$ of the periodic orbit:

$$
\phi_{T}(\mathbf{x})=\mathbf{x}_{0}+M\left(\mathbf{x}-\mathbf{x}_{0}\right)+O\left(\left\|\mathbf{x}-\mathbf{x}_{0}\right\|^{2}\right) .
$$


As the monodromy matrix $M$ is symplectic,

$$
\operatorname{Spec} M=\left\{1,1, \lambda_{1}, \lambda_{1}^{-1}, \lambda_{2}, \lambda_{2}^{-1}\right\} .
$$

The stability parameters of the periodic orbit, that are defined as $s_{j}=\lambda_{j}+\lambda_{j}^{-1}$ for $j=1,2$, can be of one of the following kinds:

- Hyperbolic: $s_{j} \in \mathbb{R},\left|s_{j}\right|>2$. It is equivalent to $\lambda_{j} \in \mathbb{R} \backslash\{-1,1\}$.

- Elliptic: $s_{j} \in \mathbb{R},\left|s_{j}\right|<2$. It is equivalent to $\lambda_{j}=e^{i \rho}$ with $\rho \in \mathbb{R}$ (if $\left|s_{j}\right|=2$, then it is said to be parabolic).

- Complex unstable: $s_{j} \in \mathbb{C} \backslash \mathbb{R}$. It is equivalent to $\lambda_{j} \in \mathbb{C} \backslash \mathbb{R},\left|\lambda_{j}\right| \neq 1$.

If $s_{j}$ is complex unstable, then $s_{3-j}$ is also complex unstable and, in fact, $s_{3-j}=\overline{s_{j}}$. If $s_{j}$ is hyperbolic, then the periodic orbit has stable and unstable manifolds, whose sections at $\mathbf{x}_{0}$ through the $\left\{\lambda_{j}, \lambda_{j}^{-1}\right\}$-eigenplane of $M$ are tangent to the $\left\{\lambda_{j}, \lambda_{j}^{-1}\right\}$-eigenvectors at $\mathbf{x}_{0}$. If $s_{j}$ is elliptic, the $\left\{\lambda_{j}, \lambda_{j}^{-1}\right\}$-eigenplane of $M$ through $\mathbf{x}_{0}$ is foliated (in the linear approximation) by invariant curves of the restriction of the linearization of $\phi_{T}$ (that is, the map $\mathbf{x} \rightarrow \mathbf{x}_{0}+M\left(\mathbf{x}-\mathbf{x}_{0}\right)$ ), which have rotation number $\rho$. For the full system, some of these invariant curves subsist and give rise to $2 \mathrm{D}$ tori.

In what follows, we will say that a periodic orbit has central part if one of the stability parameters $s_{1}, s_{2}$ is elliptic. If $\left|s_{i}\right|<2$ for $i=1,2$, the periodic orbit is linearly stable.

\section{Methodology}

This section is devoted to the development of the methodology used for the continuation of families of periodic orbits and invariant 2D tori. For the computation of families of invariant tori, the practical implementation in a parallel computer will also be discussed.

The procedure for the computation of families of both periodic orbits and invariant tori is based on a predictor-corrector scheme that will be explained in the following sections.

\subsection{Refinement and continuation of periodic orbits}

The following guidelines have been taken into account to design the methodology for the numerical refinement and continuation of periodic orbits in this section:

- As, in principle, we are not looking for periodic orbits with any kind of symmetry, the integrations will be carried out for the full period of each orbit.

- We will use multiple shooting ([18]), in order to avoid the difficulties due to the integration of very unstable orbits over large periods.

- We want to be able to continue the families of periodic orbits (p.o.) with respect to different parameters using the same routines. 


\subsubsection{The system of equations}

Consider the Hamiltonian of the RTBP, $H(\mathbf{x})$, with vector-field $X_{H}(\mathbf{x})$ and flow $\phi_{t}(\mathbf{x})$ for $\mathbf{x} \in \mathbb{R}^{6}$ so that $\frac{d}{d t} \phi_{t}(\mathbf{x})=X_{H}\left(\phi_{t}(\mathbf{x})\right)$ and $\phi_{0}(\mathbf{x})=\mathbf{x}$. Let $\{g(\mathbf{x})=0\}$ be a surface of section and $T(\mathbf{x})$ the time necessary to intersect transversally $\{g(\mathbf{x})=0\}$, in the direction of $\nabla g(\mathbf{x})$, starting at $\mathbf{x}$. Then, the system of equations used to refine $m$ initial conditions $\mathbf{x}_{0}, \ldots, \mathbf{x}_{m-1} \in \mathbb{R}^{6}$ to a periodic orbit can be written as

$$
\left\{\begin{array}{ll}
H\left(\mathbf{x}_{0}\right)-h & =0 \\
T\left(\mathbf{x}_{m-1}\right)-\frac{\tau}{m} & =0 \\
\phi_{\frac{\tau}{m}}\left(\mathbf{x}_{i}\right)-\mathbf{x}_{i+1} & =0 \\
\phi_{T\left(\mathbf{x}_{m-1}\right)}\left(\mathbf{x}_{m-1}\right)-\mathbf{x}_{0} & =0
\end{array} \quad i=0 \div m-2\right.
$$

where the multiple shooting equations $\phi_{\frac{\tau}{m}}\left(\mathbf{x}_{i}\right)-\mathbf{x}_{i+1}=0$ are assumed not to exist if $m=1$. This is a system of $6 m+2$ equations and $6 m+2$ unknowns: $h, \tau \in \mathbb{R}$ and $\mathbf{x}_{0}, \ldots, \mathbf{x}_{m-1} \in \mathbb{R}^{6}$. Note that, due to the definition of $T(\mathbf{x})$, the last equation implicitly forces $g\left(\mathbf{x}_{0}\right)=0$.

In order to implement Newton's method, the derivatives of all the above equations are needed. They can be calculated directly in terms of $X_{H}(\mathbf{x}), D \phi_{t}(\mathbf{x})$ and $D T(\mathbf{x}) . D \phi_{t}(\mathbf{x})$ can be obtained from the first variational equations, and $D T(\mathbf{x})$ can be obtained by differentiating $g\left(\phi_{T(\mathbf{x})}(\mathbf{x})\right) \equiv 0$, which gives

$$
D T(\mathbf{x})=-\frac{D g\left(\phi_{T(\mathbf{x})}(\mathbf{x})\right) D \phi_{T(\mathbf{x})}(\mathbf{x})}{D g\left(\phi_{T(\mathbf{x})}(\mathbf{x})\right) X_{H}\left(\phi_{T(\mathbf{x})}(\mathbf{x})\right)} .
$$

In all our computations the surface of section $\{g(\mathbf{x})=0\}$ is an hyperplane, so $D g\left(\phi_{T(\mathbf{x})}(\mathbf{x})\right)$ is a constant row vector.

System (2) does not need to be compatible. In fact the two first equations are not independent, because a level of energy determines uniquely a periodic orbit (locally) within a family, as well as a prescribed value of the period does. Our strategy consists in eliminating certain equations and keeping constant certain unknowns of (2) in order to perform continuations with respect to different parameters, as will be explained below. From now on, by "eliminating an unknown" we will mean keeping this unknown constant.

\subsubsection{Refinement of a periodic orbit}

To refine a p.o. we eliminate equations and unknowns of system (2) in order to get a new system of equations which has the periodic orbit as its unique solution (except in the case of bifurcation orbits). Several possibilities are:

- Refinement of a p.o. of a given energy level: we eliminate the second equation and unknowns $h, \tau$.

- Refinement of a p.o. of a given period: we eliminate the first equation and the unknowns $h, \tau$.

- Refinement of a p.o. of a given energy level and with a prescribed value of a coordinate: we eliminate the second equation and the unknowns $h$ and the prescribed coordinate. 
After elimination of equations and unknowns, we apply standard Newton's method to the system obtained. Let us denote it by $F(X)=0$, then given a first approximation $X^{(0)}$ we proceed iteratively as

$$
\begin{aligned}
D F\left(X^{(k)}\right) Y^{(k)} & =F\left(X^{(k)}\right) \\
X^{(k+1)} & =X^{(k)}-Y^{(k)},
\end{aligned}
$$

stopping at the first $k$ such that $\left\|F\left(X^{(k)}\right)\right\|$ or $\left\|X^{(k)}-X^{(k-1)}\right\|$ are under a given tolerance.

We remark that, in the three possibilities above defined, (3) is a full-rank non-square linear system. A square system could be obtained by eliminating a further equation, for instance the equation corresponding to a coordinate that is determined by the surface of section. This approach would have the drawback of being section-dependent. An alternative is to use a method for solving linear systems that does not depend on the linear system being square, for instance a QR factorization or singular value decomposition (SVD [4]). We have used SVD because it is also well suited for the continuation of periodic orbits. The loss of efficiency, in terms of computing effort, with respect to other methods is not important in this case, since we are dealing with small dimensions.

\subsubsection{Continuation of a family of p.o.}

As in any Hamiltonian system, periodic orbits are not isolated in the RTBP but embedded in families. Once we have computed a single periodic orbit, we want to continue the corresponding family. The general ideas used are those of [17].

Our strategy for the continuation consists in eliminating equations and unknowns of system (2) in order to get a new system of equations which has the family of p.o. as a solution. Several possibilities are:

- Continuation w.r.t. energy: we eliminate the 2 nd equation and $\tau$.

- Continuation w.r.t. period: we eliminate the 1st equation and $h$.

Let $F(X)=0$ be the system of equations obtained after elimination. Given a p.o., in order to predict a new p.o. of the family in which it is embedded, we consider the family as a manifold and move along its tangent space. Since this manifold is expressed by $F(X)=0$, its tangent space, at a given orbit $X$, is given by $\operatorname{Ker} D F(X)$. Therefore, we proceed in the following manner: given $X^{(k)}$

- We compute

$$
Y^{(k)}=X^{(k)}+\delta V^{(k)}
$$

being $\delta \in \mathbb{R}, V^{(k)} \in \operatorname{Ker} D F\left(X^{(k)}\right)$ and $\left\|V^{(k)}\right\|_{2}=1$.

- We refine $Y^{(k)}=: Y^{(k, 0)}$ using Newton's method:

$$
\begin{aligned}
D F\left(Y^{(k, j)}\right) Z^{(j)} & =F\left(Y^{(k, j)}\right) \\
Y^{(k, j+1)} & =Y^{(k, j)}-Z^{(j)}
\end{aligned}
$$

- We take as new member of the family, $X^{(k+1)}$, the first $Y^{(k, j)}$ such that $\left\|F\left(Y^{(k, j)}\right)\right\|$ or $\left\|Y^{(k, j)}-Y^{(k, j-1)}\right\|$ is under a given tolerance. 
The continuation step, $\delta$, is corrected during the process in order to keep constant the number of iterates necessary for refinement.

Note that, because $F(X)=0$ has the family of p.o. as solution, the linear system (5) has not unique solution but a 1-dimensional kernel. When solving (5) we choose the minimum Euclidean norm solution, in order to find the "closest" p.o. of the family. In the computations we have used SVD, because it directly gives both the kernel of $D F\left(X^{(k)}\right)$ for computing $V^{(k)}$ and the minimum-norm solution of (5).

An additional comment is needed for the continuation w.r.t. energy using multiple shooting $(m>1)$. The unknown $\tau$, which is kept fixed, does not need to be the period of the p.o., but it should be close, in order to get the conditions $\mathbf{x}_{0}, \ldots, \mathbf{x}_{m-1}$ well spaced along the orbit. Thus it is advisable to set $\tau$ equal to the period of the p.o. after each continuation step. This implies to correct $\mathbf{x}_{1}, \ldots, \mathbf{x}_{m-1}$ in order to satisfy $F(X)=0$ again.

\subsection{Refinement and continuation of invariant tori}

The procedure used here for the refinement and continuation of invariant tori is based on looking for the Fourier series of the parametrization of an invariant curve on a torus, asking numerically for quasi-periodic motion. This kind of procedures have been introduced in [3].

Concretely, let $H(\mathbf{x}), X_{H}(\mathbf{x})$ and $\phi_{t}(\mathbf{x})$ be as above. We can look for a parametrization of a 2-dimensional torus $\psi: \mathbb{T}^{2}=\mathbb{R}^{2} / 2 \pi \mathbb{Z} \rightarrow \mathbb{R}^{n}(n=6$ for the RTBP), satisfying

$$
\psi(\theta+\omega t)=\phi_{t}(\psi(\theta)), \quad \forall \theta \in \mathbb{T}^{2}, \forall t \in \mathbb{R},
$$

where $\omega=\left(\omega_{1}, \omega_{2}\right) \in \mathbb{R}^{2}$ is the vector of frequencies of the torus. Let us denote by $T_{i}$ the period corresponding to the $\omega_{i}$ frequency, that is $T_{i}=2 \pi / \omega_{i}$, and $\theta=(\xi, \eta)$.

In order to reduce the dimension of the problem, instead of looking for the parametrization of the whole torus, we can look for the parametrization of a curve $\left\{\eta=\eta_{0}\right\}$ (or $\left\{\xi=\xi_{0}\right\}$ ) on the torus, which is invariant under $\phi_{T_{2}}$, namely

$$
\phi_{T_{2}}\left(\psi\left(\xi, \eta_{0}\right)\right)=\psi\left(\xi+\omega_{1} T_{2}, \eta_{0}\right), \quad \forall \xi \in \mathbb{T}^{1}
$$

Then, we look for a parametrization $\varphi: \mathbb{T}^{1} \rightarrow \mathbb{R}^{n}$ satisfying

$$
\varphi(\xi+\rho)=\phi_{\delta}(\varphi(\xi)), \quad \forall \xi \in \mathbb{T}^{1},
$$

where $\delta=T_{2}$ and $\rho=\delta \omega_{1}$. Note that $\rho$ is the rotation number of the curve we are looking for.

The original two-dimensional parametrization of the whole torus can be recovered from a parametrization of the invariant curve $\left\{\eta=\eta_{0}\right\}$. This is because (6) allows to reach any point of the torus integrating from a suitable point of the invariant curve, namely

$$
\psi(\xi, \eta)=\phi_{\frac{\eta-\eta_{0}}{2 \pi} \delta}\left(\varphi\left(\xi-\frac{\eta-\eta_{0}}{2 \pi} \rho\right)\right) .
$$

In fact, given a parametrization $\varphi$ of an invariant curve satisfying (8), then equation (9) defines a 2 D parametrization of a $2 \mathrm{D}$ torus containing the invariant curve $\{\varphi(\xi)\}_{\xi \in \mathbb{T}^{1}}$ and satisfying $(6)$, as shown in the following 
Proposition 2.1 The function $\psi$ defined as in (9), where $\varphi$ is assumed to satisfy (8), is $2 \pi-$ periodic in each component and satisfies

$$
\phi_{t}(\psi(\xi, \eta))=\psi\left((\xi, \eta)+t\left(\frac{\rho}{\delta}, \frac{2 \pi}{\delta}\right)\right)
$$

Proof: The $2 \pi$-periodicity in $\xi$ comes from the $2 \pi$-periodicity of $\varphi$. With respect to the $2 \pi-$ periodicity in $\eta$, we have

$$
\begin{gathered}
\psi(\xi, \eta+2 \pi)=\phi_{\frac{\eta-\eta_{0}}{2 \pi} \delta+\delta}\left(\varphi\left(\xi-\frac{\eta-\eta_{0}}{2 \pi} \rho-\rho\right)\right)=\phi_{\frac{\eta-\eta_{0}}{2 \pi} \delta}\left(\phi_{\delta}\left(\varphi\left(\xi-\frac{\eta-\eta_{0}}{2 \pi} \rho-\rho\right)\right)\right) \\
\stackrel{(8)}{=} \phi_{\frac{\eta-\eta_{0}}{2 \pi} \delta}\left(\varphi\left(\xi-\frac{\eta-\eta_{0}}{2 \pi} \rho\right)\right)=\psi(\xi, \eta) .
\end{gathered}
$$

Finally,

$$
\begin{aligned}
\phi_{t}(\psi(\xi, \eta)) & =\phi_{\frac{\eta-\eta_{0}}{2 \pi} \delta+t}\left(\varphi\left(\xi-\frac{\eta-\eta_{0}}{2 \pi} \rho\right)\right) \\
& =\phi_{\frac{\eta+t \frac{2 \pi}{\delta}-\eta_{0}}{2 \pi} \delta}\left(\varphi\left(\xi-\frac{\eta+t \frac{2 \pi}{\delta}-\eta_{0}}{2 \pi} \rho+t \frac{\rho}{\delta}\right)\right) \\
& =\psi\left(\xi+t \frac{\rho}{\delta}, \eta+t \frac{2 \pi}{\delta}\right),
\end{aligned}
$$

and this ends the proof.

Note that if equation (8) is satisfied for a value of $\rho$, it is also satisfied if we replace $\rho$ by $\rho+2 \pi j, j \in \mathbb{Z}$. From (10), this gives a frequency vector $((\rho+2 \pi j) / \delta, 2 \pi / \delta)$, which is different from $(\rho / \delta, 2 \pi / \delta)$. This is a particular case of the following

Proposition 2.2 Assume that $\psi: \mathbb{T}^{d} \rightarrow \mathbb{R}^{n}$ is a parametrization of a d-dimensional torus such that there exists a vector $\omega \in \mathbb{R}^{d}$ of rationally independent frequencies (that is, $k \in \mathbb{Z}^{d}$, $k \cdot \omega=0 \Rightarrow k=0$, where $\cdot$ denotes the Euclidean scalar product) verifying

$$
\phi_{t}(\psi(\theta))=\psi(\theta+t \omega), \quad \forall \theta \in \mathbb{T}^{d}, \quad \forall t \in \mathbb{R} .
$$

Let $A$ be a $d \times d$ unimodular matrix (that is, with integer coefficients and determinant \pm 1 ) and define $\nu=A \omega$. Then there exists a parametrization $\widetilde{\psi}: \mathbb{T}^{d} \rightarrow \mathbb{R}^{n}$ of the same torus (i.e. $\{\psi(\theta)\}_{\theta \in \mathbb{T}^{d}}=\{\widetilde{\psi}(\theta)\}_{\theta \in \mathbb{T}^{d}}$ ) satisfying

$$
\phi_{t}(\widetilde{\psi}(\theta))=\widetilde{\psi}(\theta+t \nu), \quad \forall \theta \in \mathbb{T}^{d}, \quad \forall t \in \mathbb{R} .
$$

Proof: Assume $\psi(\theta)=\sum_{k \in \mathbb{Z}^{d}} a_{k} e^{i k \cdot \theta}$. Then,

$$
\psi(\omega t)=\sum_{k \in \mathbb{Z}^{d}} a_{A^{\top} k} e^{i\left(A^{\top} k\right)^{\top} \omega t}=\sum_{k \in \mathbb{Z}^{d}} a_{A^{\top} k} e^{i k^{\top}(A \omega) t}=\widetilde{\psi}(\nu t),
$$

being

$$
\widetilde{\psi}(\theta)=\sum_{k \in \mathbb{Z}^{d}} a_{A^{\top} k} e^{i k \cdot \theta} .
$$


Then, for any $t \in \mathbb{R}$,

$$
\widetilde{\psi}(\nu t)=\psi(\omega t)=\phi_{t}(\psi(0))=\phi_{t}(\widetilde{\psi}(0))
$$

Now let $\theta \in[0,2 \pi]^{d}$ and let $\left\{s_{n}\right\}_{n \in \mathbb{N}} \subset \mathbb{R}$ with $\left(s_{n} \nu\right) \% 2 \pi \stackrel{n \rightarrow \infty}{\longrightarrow} \theta$ (such a sequence exists because of the rational independence of $\omega)$, where we denote

$$
\left(\theta_{1}, \ldots, \theta_{d}\right) \% 2 \pi=\left(\theta_{1}-2 \pi\left[\theta_{1} /(2 \pi)\right], \ldots, \theta_{d}-2 \pi\left[\theta_{d} /(2 \pi)\right]\right)
$$

and [ ] denotes integer part. Then, for any $t \in \mathbb{R}$, using the continuity and $2 \pi$-periodicity in each argument of $\widetilde{\psi}$, we have

$$
\begin{aligned}
\widetilde{\psi}(\theta+t \nu) & =\lim _{n \rightarrow \infty} \widetilde{\psi}\left(s_{n} \nu+t \nu\right) \stackrel{(11)}{=} \lim _{n \rightarrow \infty} \phi_{t}\left(\phi_{s_{n}}(\widetilde{\psi}(0))\right) \\
& \stackrel{(11)}{=} \lim _{n \rightarrow \infty} \phi_{t}\left(\widetilde{\psi}\left(s_{n} \nu\right)\right)=\phi_{t}\left(\widetilde{\psi}\left(\lim _{n \rightarrow \infty} s_{n} \nu \% 2 \pi\right)\right) \\
& =\phi_{t}(\widetilde{\psi}(\theta))
\end{aligned}
$$

which ends the proof.

\subsubsection{Indeterminations of the Fourier representation}

We will look for $\varphi$ as a truncated Fourier series,

$$
\varphi(\xi)=A_{0}+\sum_{k=1}^{N_{f}}\left(A_{k} \cos (k \xi)+B_{k} \sin (k \xi)\right),
$$

with $A_{k}, B_{k} \in \mathbb{R}^{n}$. This representation of the geometrical torus $\{\psi(\theta)\}_{\theta \in \mathbb{T}^{2}}$ is non unique for two reasons:

- For each choice of $\eta_{0}$ we have a different $\varphi$ in (12), i.e., a different invariant curve on the torus $\{\psi(\theta)\}_{\theta \in \mathbb{T}^{2}}$.

- Given the parametrization (12), for each $\xi_{0} \in \mathbb{T}^{1}, \varphi\left(\xi-\xi_{0}\right)$ is a different parametrization with a different Fourier expansion of the same invariant curve $\{\varphi(\xi)\}_{\xi \in \mathbb{T}^{1}}$ of the torus $\{\psi(\theta)\}_{\theta \in \mathbb{T}^{2}}$. Indeed, its Fourier expansion is

$$
\varphi\left(\xi-\xi_{0}\right)=A_{0}+\sum_{k=1}^{N_{f}}\left(\widetilde{A}_{k} \cos (k \xi)+\widetilde{B}_{k} \sin (k \xi)\right),
$$

being

$$
\begin{aligned}
& \widetilde{A}_{k}=A_{k} \cos \left(k \xi_{0}\right)-B_{k} \sin \left(k \xi_{0}\right) \\
& \widetilde{B}_{k}=A_{k} \sin \left(k \xi_{0}\right)+B_{k} \cos \left(k \xi_{0}\right) .
\end{aligned}
$$

We will call this "coefficient modulation", in analogy with the terminology used in signal processing 
Our approach to overcome the first indetermination has been to keep constant (i.e. to eliminate as unknown) one coordinate of $A_{0}$. Care must be taken to verify that this condition is valid along the continuation of the tori.

As for the second indetermination, we remark that, for any $k \geq 1$, if $\left(A_{k}^{j}, B_{k}^{j}\right) \neq(0,0)$ (here $A_{k}^{j}$ denotes the $j$-th coordinate of $A_{k}$ ), from (13) there exists a $\xi_{0}$ that gives $\widetilde{A}_{k}^{j}=0$. Thus by setting $A_{k}^{j}=0$ we will avoid the indetermination, as long as $B_{k}^{j}$ remains different from zero. Usually we have taken $k=1$ and $j$ has been selected in order to maximize $\left\|\left(A_{1}^{j}, B_{1}^{j}\right)\right\|_{2}$. In order to not to depend on $B_{k}^{j}$ being different from zero, an alternative strategy is described in section 2.2.5.

\subsubsection{Multiple shooting}

In order to deal with high instability, we will use a multiple shooting procedure. It consists in looking for several invariant curves on the torus $\{\psi(\theta)\}_{\theta \in \mathbb{T}^{d}}$ instead of just one, in order to reduce the maximum time of integration to a fraction of $\delta$. Concretely, we will look for $m$ parametrizations $\varphi_{0}=\varphi, \varphi_{1}, \ldots, \varphi_{m-1}$ satisfying

$$
\left\{\begin{aligned}
\varphi_{j+1}(\xi) & =\phi_{\frac{\delta}{m}}\left(\varphi_{j}(\xi)\right) \quad(j=0 \div m-2) \\
\varphi_{0}(\xi+\rho) & =\phi_{\frac{\delta}{m}}\left(\varphi_{m-1}(\xi)\right)
\end{aligned}\right.
$$

for all $\xi \in \mathbb{T}^{1}$.

\subsubsection{The system of equations}

Following [3], we will turn (14) into a finite system of equations by discretizing $\mathbb{T}^{1}$ in $2 N_{f}+1$ values of $\xi$. We will simply take

$$
\xi_{i}=i \frac{2 \pi}{1+2 N_{f}} \quad\left(i=0 \div 2 N_{f}\right)
$$

In addition to the Fourier coefficients, we have to consider $\delta, \rho$ as unknowns since they are expected to vary along continuations. In order to describe the tori in terms of energy, we will add a new unknown $h$ and a new equation $H\left(\varphi_{0}(0)\right)-h=0$, which will prescribe an energy level for the torus being computed.

In order to simplify the notation, let us denote the Fourier coefficients as

$$
\mathcal{F}=\left(A_{0}, A_{1}, B_{1}, \ldots, A_{N_{f}}, B_{N_{f}}\right)
$$

and

$$
\Psi(\xi, \mathcal{F})=A_{0}+\sum_{k=1}^{N_{f}}\left(A_{k} \cos (k \xi)+B_{k} \sin (k \xi)\right) .
$$

Then, the system of equations to be solved is

$$
\begin{aligned}
& H\left(\Psi\left(0, \mathcal{F}_{0}\right)\right)-h=0 \\
& \Psi\left(\xi_{i}, \mathcal{F}_{j+1}\right)-\phi_{\frac{\delta}{m}}\left(\Psi\left(\xi_{i}, \mathcal{F}_{j}\right)\right)=0 \quad j=0 \div m-2, i=0 \div 2 N_{f} \\
& \Psi\left(\xi_{i}+\rho, \mathcal{F}_{0}\right)-\phi_{\frac{\delta}{m}}\left(\stackrel{m}{\Psi}\left(\xi_{i}, \mathcal{F}_{m-1}\right)\right)=0 \quad i=0 \div 2 N_{f},
\end{aligned}
$$

where the unknowns are $h, \delta, \rho, \mathcal{F}_{0}, \ldots, \mathcal{F}_{m-1} . \mathcal{F}_{i}$ stands for the Fourier coefficients of the curve $\varphi_{i}$ in the multiple shooting (14). As in the case of periodic orbits, the multiple shooting equations $\Psi\left(\xi_{i}, \mathcal{F}_{j+1}\right)-\phi_{\frac{\delta}{m}}\left(\Psi\left(\xi_{i}, \mathcal{F}_{j}\right)\right)=0$ do not exist if $m=1$. 


\subsubsection{Refinement of an invariant torus}

In order to refine an invariant torus, we first eliminate two unknowns in system (16). With this, we set an invariant curve on the torus and avoid coefficient modulation. After that we eliminate two more unknowns in order to have the torus we are looking for as a unique solution of our system of equations. This is because the tori we will compute are always embedded in two-parametric Cantorian families. A concrete example, with the actual unknowns eliminated, will be given at the beginning of section 3.3 .

After elimination of these four unknowns, we use Newton's method to the system obtained as in the case of periodic orbits. Equation (3) applies if we denote this system by $F(X)=0$. As for the numerical method used to solve (2), we have used QR decomposition instead of SVD in this case, due to efficiency reasons. Additional details will be given in section 2.4.2.

\subsubsection{Continuation of a family of tori}

We perform the continuation of families of invariant tori using a predictor-corrector scheme. The idea is the same as in the case of periodic orbits: given a torus, in order to predict a new torus of the family, we consider the family as a manifold of invariant tori and move along its tangent space. Since this manifold is expressed as $F(X)=0$, its tangent space at a given torus $X$ is given by $\operatorname{Ker} D F(X)$. Note that this is not strictly true, since the manifolds of invariant tori are Cantorian, but it works in practice as long as the "gaps" due to resonances are not too large.

Therefore, we proceed as in the case of p.o.: given a torus of the family $X^{(k)}$, we predict a new one as $Y^{(k)}=X^{(k)}+\delta V^{(k)}$ where $\left\|V^{(k)}\right\|_{2}=1, V^{(k)} \in \operatorname{Ker} D F\left(X^{(k)}\right)$, and then we correct it by Newton's method.

Due to the indetermination introduced by coefficient modulation, we will use different systems of equations for prediction and correction. The system we use for prediction is obtained from (16) by eliminating two unknowns, one in order to set an invariant curve on the torus and another in order to fix a one-parametric family (our families of tori are two-parametric). Let us denote this system as $F_{p}(X)=0$. Then $\operatorname{Ker} D F_{p}(X)$ is two-dimensional and includes coefficient modulation. We choose $V^{(k)} \in \operatorname{Ker} D F_{p}(X)$ orthogonal to the direction corresponding to coefficient modulation, which can be obtained by differentiating (13) with respect to $\xi_{0}$.

In order to perform the correction, we will remove from $F_{p}(X)=0$ an additional unknown, which will be chosen according to the following criteria:

- We will eliminate $A_{1}^{J}$ or $B_{1}^{J}$, where $J$ is such that $\left\|\left(A_{1}^{J}, B_{1}^{J}\right)\right\|_{2}=\max _{i=1 \div n}\left\|\left(A_{1}^{i}, B_{1}^{i}\right)\right\|_{2}$. The reason for this choice is heuristic and tries to prevent levying conditions on harmonics of low amplitude.

- Between $A_{1}^{J}$ and $B_{1}^{J}$, we select the coordinate for which $\min \left(\left|A_{1}^{J}\right|,\left|B_{1}^{J}\right|\right)$ is achieved. This avoids situations like the following: assume that, before the correction, $A_{1}^{J}=0$ and $\left|B_{1}^{J}\right|=\alpha$. If we eliminate $B_{1}^{J}$, we are forcing $\left\|\left(A_{1}^{J}, B_{1}^{J}\right)\right\|_{2}>\alpha$ and therefore we will not be able to continue if $\left\|\left(A_{1}^{J}, B_{1}^{J}\right)\right\|_{2}$ decreases along the family. If $\left|A_{1}^{J}\right|$ is different from zero but small, we will be able to continue but the continuation step $\delta$ will be artificially reduced.

We prefer this strategy for coefficient modulation instead of the one pointed out in 2.2.1 in order to not to depend on a Fourier coefficient being different from zero. 
As in the case of periodic orbits, the linear system solved during correction is rank-deficient. We solve it using QR with column pivoting instead of SVD for efficiency reasons. Additional details will be given in sections 2.4.2 and 2.4.3.

\subsubsection{Error estimation}

In order to have some control over the error due to the discretization of $\mathbb{T}^{1}$, we proceed as in [3]: we evaluate (16) over a finer discretization than (15) and increase the number of harmonics until the maximum norm is under a given tolerance. That is, we increase $N_{f}$ until

$$
\max _{\substack{i=0 \div 2 N_{f}, j=0 \div m-2 \\
k=0 \div M\left(1+2 N_{f}\right)-1}}\left\|\begin{array}{c}
\Psi\left(\widetilde{\theta}_{k}, \mathcal{F}_{j+1}\right)-\phi_{\frac{\delta}{m}}\left(\Psi\left(\widetilde{\theta}_{k}, \mathcal{F}_{j}\right)\right) \\
\Psi\left(\widetilde{\theta}_{k}+\rho, \mathcal{F}_{0}\right)-\phi_{\frac{\delta}{m}}\left(\Psi\left(\widetilde{\theta}_{k}, \mathcal{F}_{m-1}\right)\right)
\end{array}\right\|_{\infty}<t o l
$$

being $\widetilde{\theta}_{k}=k \frac{2 \pi}{M\left(1+2 N_{f}\right)}$. We have typically used $t o l=10^{-10}$ and $M=50$.

\subsection{Starting the continuation of tori from the central part of a peri- odic orbit}

Given a periodic orbit with central part, we use the linear approximation of the flow in order to get an initial seed to start the computation of an invariant torus around this orbit.

Let $\mathbf{x}_{0}$ be an initial condition of a periodic orbit of period $T$ so that $\phi_{T}\left(\mathbf{x}_{0}\right)=\mathbf{x}_{0}$. We denote the linearized time $t$ flow around $\mathbf{y}$ by

$$
L_{\phi_{t}}^{\mathbf{y}}(\mathbf{x})=\phi_{t}(\mathbf{y})+D \phi_{t}(\mathbf{y})(\mathbf{x}-\mathbf{y}) .
$$

Let $\cos \nu+i \sin \nu$ be an eigenvalue of the monodromy matrix of the periodic orbit, $M=D \phi_{T}\left(\mathbf{x}_{0}\right)$, and let $\mathbf{v}_{1}+i \mathbf{v}_{2}$ be an associated eigenvector. Define $L_{\varphi}$ as

$$
\begin{aligned}
L_{\varphi}(\xi)=\mathbf{x}_{0} & +\gamma\left(\mathbf{v}_{1} \cos \xi_{0}+\mathbf{v}_{2} \sin \xi_{0}\right) \cos \xi \\
& +\gamma\left(\mathbf{v}_{1} \sin \xi_{0}-\mathbf{v}_{2} \cos \xi_{0}\right) \sin \xi,
\end{aligned}
$$

where $\gamma \in \mathbb{R}$ is a parameter. Then we have the following

Proposition 2.3 For all $\xi \in[0,2 \pi], L_{\varphi}$ verifies

$$
L_{\varphi}(\xi+\nu)-L_{\phi_{T}}^{\mathbf{x}_{0}}\left(L_{\varphi}(\xi)\right)=0 .
$$

Proof: Since $\mathbf{v}_{1}+i \mathbf{v}_{2} \in \operatorname{Spec} M$, we have

$$
\begin{aligned}
M\left(\mathbf{v}_{1}+i \mathbf{v}_{2}\right) & =(\cos \nu+i \sin \nu)\left(\mathbf{v}_{1}+i \mathbf{v}_{2}\right) \\
& =\left(\mathbf{v}_{1} \cos \nu-\mathbf{v}_{2} \sin \nu\right)+i\left(\mathbf{v}_{1} \sin \nu+\mathbf{v}_{2} \cos \nu\right)
\end{aligned}
$$

which can be rewritten as

$$
M\left[\mathbf{v}_{1}, \mathbf{v}_{2}\right]=\left[\mathbf{v}_{1}, \mathbf{v}_{2}\right]\left(\begin{array}{cc}
\cos \nu & \sin \nu \\
-\sin \nu & \cos \nu
\end{array}\right)=\left[\mathbf{v}_{1}, \mathbf{v}_{2}\right] R_{\nu}
$$


where $\left[\mathbf{v}_{1}, \mathbf{v}_{2}\right]$ denotes the $6 \times 2$ matrix with columns $\mathbf{v}_{1}, \mathbf{v}_{2}$ and

$$
R_{\xi}=\left(\begin{array}{cc}
\cos \xi & \sin \xi \\
-\sin \xi & \cos \xi
\end{array}\right)
$$

is the rotation of the plane of angle $\xi$. Now,

$$
\begin{aligned}
L_{\phi_{T}}^{\mathbf{x}_{0}}\left(L_{\varphi}(\xi)\right) & =\mathbf{x}_{0}+M\left[\mathbf{v}_{1}, \mathbf{v}_{2}\right]\left(\begin{array}{cc}
\cos \left(\xi-\xi_{0}\right) & \sin \left(\xi-\xi_{0}\right) \\
-\sin \left(\xi-\xi_{0}\right) & \cos \left(\xi-\xi_{0}\right)
\end{array}\right)\left(\begin{array}{l}
\gamma \\
0
\end{array}\right) \\
& =\mathbf{x}_{0}+M\left[\mathbf{v}_{1}, \mathbf{v}_{2}\right] R_{\xi-\xi_{0}}\left(\begin{array}{l}
\gamma \\
0
\end{array}\right) \stackrel{(20)}{=} \mathbf{x}_{0}+\left[\mathbf{v}_{1}, \mathbf{v}_{2}\right] R_{\nu} R_{\xi-\xi_{0}}\left(\begin{array}{l}
\gamma \\
0
\end{array}\right) \\
& =\mathbf{x}_{0}+M\left[\mathbf{v}_{1}, \mathbf{v}_{2}\right] R_{\xi+\nu-\xi_{0}}\left(\begin{array}{l}
\gamma \\
0
\end{array}\right)=L_{\varphi}(\xi+\nu),
\end{aligned}
$$

as the proposition states.

Note that equation (19) is the linearized version of (8). We can therefore use (9) to construct the one-parametric family, with parameter $\gamma$, of $2 \mathrm{D}$ tori invariant by the linear approximation of the flow around the periodic orbit, namely

$$
L_{\psi}(\xi, \eta)=L_{\phi_{\frac{\eta}{2 \pi} T}^{\mathbf{x}_{0}}}^{\mathbf{n}_{\varphi}}\left(L_{\varphi}\left(\xi-\frac{\eta}{2 \pi} \nu\right)\right)
$$

Doing similar calculations to the ones of Proposition 2.1, it can be verified that

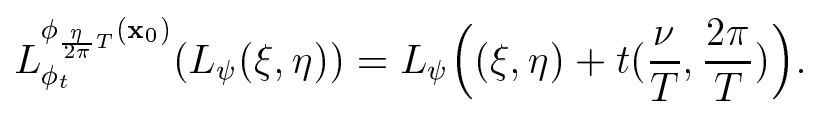

Therefore, we expect to find in a neighborhood of the periodic orbit $2 \mathrm{D}$ invariant tori with frequency vector close to $(\nu / T, 2 \pi / T)$.

Note that, since $e^{ \pm i \nu} \in \operatorname{Spec} M, \nu$ is determined up to its sign and an integer multiple of $2 \pi$. This gives for the frequency vector the following possibilities:

$$
\left(\frac{ \pm \nu+2 \pi j}{T}, \frac{2 \pi}{T}\right), \quad j \in \mathbb{Z}
$$

which, according to Proposition 2.2, are unimodular transformations of the initial frequency vector, namely

$$
\left(\begin{array}{c}
\frac{ \pm \nu+2 \pi j}{T} \\
\frac{2 \pi}{T}
\end{array}\right)=\left(\begin{array}{cc} 
\pm 1 & j \\
0 & 1
\end{array}\right)\left(\begin{array}{c}
\frac{\nu}{T} \\
\frac{2 \pi}{T}
\end{array}\right)
$$

With this, what we have are invariant tori for the linear flow around the periodic orbit. We will use these tori to get an initial seed for the procedure.

\subsubsection{Starting "longitudinally" to the periodic orbit}

By this we mean to look for an initial torus integrating "along" the periodic orbit, that is, taking $\delta$ in (8) close to the period of the periodic orbit. We will therefore look for an invariant curve 
on this initial torus close to $\left\{L_{\psi}(\xi, 0)\right\}_{\xi \in \mathbb{T}^{1}}$. From equations (21), (19) and (18), we will take as initial seed

$$
\begin{array}{rlrl}
h & =H\left(\mathbf{x}_{0}\right), & A_{0} & =\mathbf{x}_{0}, \\
\delta & =T, & A_{1} & =\gamma\left(\mathbf{v}_{1} \cos \xi_{0}+\mathbf{v}_{2} \sin \xi_{0}\right), \\
\rho & =\nu, & B_{1} & =\gamma\left(\mathbf{v}_{1} \sin \xi_{0}-\mathbf{v}_{2} \cos \xi_{0}\right), \\
A_{j}, B_{j} & =0 \quad(j \geq 2)
\end{array}
$$

If multiple shooting is used, then initial approximations of the Fourier coefficients of the remaining invariant curves can be taken from

$$
\begin{aligned}
L_{\phi_{j \frac{T}{m}}}^{\mathbf{x}_{0}}\left(L_{\varphi}(\xi)\right)=\phi_{j \frac{T}{m}}\left(\mathbf{x}_{0}\right) & +\gamma\left(\mathbf{w}_{1}^{j} \cos \xi_{0}+\mathbf{w}_{2}^{j} \sin \xi_{0}\right) \cos \xi \\
& +\gamma\left(\mathbf{w}_{2}^{j} \sin \xi_{0}-\mathbf{w}_{2}^{j} \cos \xi_{0}\right) \sin \xi
\end{aligned}
$$

where $\mathbf{w}_{i}^{j}=D \phi_{j \frac{T}{m}}\left(\mathbf{x}_{0}\right) \mathbf{v}_{i}$.

Note that $\xi_{0}$ is free. It can be used to set to zero the coordinate we want to eliminate in order to prevent coefficient modulation.

If, during the refinement of the initial seed (23), we keep constant one nonzero coordinate of $A_{1}$ or $B_{1}$, we will avoid "falling back" to the periodic orbit, since it will no longer be a solution of our system of equations. In particular, the unknowns $\delta$ and $\rho$, which were originally set equal to the values of the periodic orbit, will be corrected to the values of the torus being refined. This avoids the need to know a priori the behavior (increasing or decreasing) of $\rho$ when we start the computation of the tori at a given periodic orbit.

\subsubsection{Starting "transversally" to the periodic orbit}

By this we mean to look for an initial torus integrating "across" the periodic orbit, that is taking $\delta$ in (8) close to the normal period of the p.o. that gives central part. We will therefore look for an invariant curve on this initial torus close to $\left\{L_{\psi}(0, \eta)\right\}_{\eta \in \mathbb{T}^{1}}$ (in particular, to the p.o.), so we will use as initial seed for the Fourier coefficients $A_{0}, A_{1}, B_{1}, \ldots$ the Fourier expansion of

$$
L_{\psi}(0, \eta)=L_{\phi_{\frac{\eta}{2 \pi} T}}\left(L_{\varphi}\left(-\frac{\eta}{2 \pi} \nu\right)\right)
$$

which can be efficiently computed from the FFT of $\left\{L_{\psi}\left(0, \frac{2 \pi j}{N}\right)\right\}_{j=0}^{N-1}$ for a suitable $N$. If multiple shooting is used, we take the Fourier coefficients of $\varphi_{j}$ from a Fourier expansion of $\phi_{j \frac{\delta}{m}}\left(L_{\psi}(0, \eta)\right)$.

The initial value for $\delta$ is taken as the period associated to the first frequency in $(22)$

$$
\delta=\frac{2 \pi}{ \pm \nu+2 \pi j} T,
$$

and, following the deduction of (8) from (7) (but exchanging the role of the two frequencies), the value for $\rho$ is taken as $\delta$ times the second frequency in (22), that is

$$
\rho=\delta \frac{2 \pi}{T}=\frac{(2 \pi)^{2}}{ \pm \nu+2 \pi j} .
$$




\subsection{Computational aspects}

In this section we will give some details of the actual implementation of the above methodology. We will focus on the continuation of families of invariant tori, since it is the critical part in terms of computing time.

\subsubsection{Continuation of a 1-parametric family of tori}

The first step in the continuation of a family of invariant tori is to get an initial seed by starting either longitudinally or transversally to a periodic orbit, as was described in section 2.3. A typical value used for the parameter $\gamma$ has been $10^{-4}$. This initial seed, which corresponds to an invariant torus of the linear flow around the periodic orbit, is refined to an invariant curve of a torus of the actual flow following section 2.2.4.

Once we have an initial torus of the family, we choose a small initial continuation step $\delta$ and proceed iteratively as follows:

1. We predict a new torus of the family following section 2.2.5.

2. We correct the predicted torus according to section 2.2.5. If the correction fails, we divide by 2 the continuation step and go to step 1.

3. We check the angle formed by the three last tori (to compute this angle we take into account $h, \delta, \rho$ and the Fourier coefficients of order zero). If it is greater than a given tolerance (we have typically used 15 degrees), we divide by 2 the continuation step and go to step 1 . In some situations, it is necessary to restart the process from the first of the three last tori.

4. We check the error bound condition (17) and, if it is not satisfied, we increase $N_{f}$, set to zero the new Fourier coefficients and go to step 2.

5. We correct the continuation step $\delta$ in order to keep the number of Newton iterations needed in step 2 within a given interval.

The continuation is stopped if one of the following conditions is true:

- The amplitude of the harmonics of order $\geq 1$ becomes zero. This means we have fallen longitudinally to a periodic orbit.

- In step $4, N_{f}$ reaches the maximum allowable value without satisfying the bound condition (17).

- In steps 2,3 or 5 , the continuation step is reduced to a value under a minimum.

Note that we do not take into account possible bifurcations.

\subsubsection{On the computing effort}

The computing effort necessary for the refinement and continuation of a family of invariant tori is distributed in three main blocks: 
- The evaluation of the system of nonlinear equations (16) and its differential. This involves the numerical integration of $1+2 N_{f}$ initial conditions through the flow of the system of differential equations formed by the RTBP equations (1) and its first variational equations.

- The solution of linear systems with the differential of the system of equations (16) as coefficient matrix. This applies to the refinement of a single invariant torus (section 2.2.4) and both to prediction and refinement in the continuation of an one-parametric family (section 2.2.5).

- The computation of the error bound (17). This involves the numerical integration of $M(1+$ $2 N_{f}$ ) initial conditions through the flow of the RTBP equations (1).

As $N_{f}$ grows, the dominant part of the computing time is expended in the solution of linear systems.

In the case of refinement of a single torus (sec. 2.2.4), the linear system corresponding to Newton's method is $\left(1+m\left(1+2 N_{f}\right) n\right) \times\left(m\left(1+2 N_{f}\right) n-1\right)$ and is full-rank. As it has been said, we use a standard QR algorithm ([4]) for solving it. In the case of continuation (sec. 2.2.5), the linear system corresponding to prediction is $\left(1+m\left(1+2 N_{f}\right) n\right) \times\left(1+m\left(1+2 N_{f}\right)\right)$, and the one corresponding to Newton's method with minimum-norm correction is $\left(1+m\left(1+2 N_{f}\right) n\right) \times$ $m\left(1+2 N_{f}\right)$. Both systems are rank-deficient. We have used QR with column pivoting [4] in these two cases. SVD can also be used, but QR with column pivoting is much faster. Concretely, for an $M \times N$ system of rank $R$, SVD requires $14 M N^{2}+8 N^{3}$ operations (following [4], we count one sum plus one product as two operations), whereas $\mathrm{QR}$ with column pivoting requires $4 M N R-2 R^{2}(M+N)+4 R^{3} / 3$. For $M=N=R$, this is a factor 16.5 .

As a timing example, if we want to continue a torus using multiple shooting with $m=2$ and $N_{f}=100$ (which has been upper limit for most of the explorations), the linear system we have to solve at each Newton iteration is $2413 \times 2411$. Every Newton iteration for these dimensions takes us 613 seconds on an Intel Pentium III at $500 \mathrm{MHz}$.

\subsubsection{Computation of kernel and minimum-norm corrections using QR with column pivoting}

Let $A x=b$ be an $m \times n$ linear system of equations with $m \geq n$ and $\operatorname{rank} A=r<n$. We are interested in computing $\operatorname{Ker} A$ and the minimum-norm solution of $A x=b$ which we will denote as $x_{L S}$.

If we apply QR factorization with column pivoting to $A$ [4], we will obtain

$$
Q^{\top} A \Pi=\left(\begin{array}{cc}
R_{11} & R_{12} \\
0 & 0
\end{array}\right)
$$

where $\Pi$ is an $n \times n$ permutation matrix, $R_{11}$ is an $r \times r$ upper triangular matrix and $R_{12}$ is an $r \times(n-r)$ matrix. Then, if we denote $\Pi^{\top} x=(y, z)^{\top}$ and $Q^{\top} b=(c, d)^{\top}$, the general least-squares solution of $A x=b$ is given by (see [4], p. 259)

$$
\Pi^{\top} x=\left\{\left(\begin{array}{c}
y: R_{11} y=c-R_{12} z \\
z
\end{array}\right)\right\}_{x \in \mathbb{R}^{n-r}} .
$$


At this point, a complete orthogonal factorization ([4]) would directly give us $x_{L S}$, but since we also need $\operatorname{Ker} A$ we have followed a different approach. Let $x_{B}$ be a particular least-squares solution of $A x=b$, which can be obtained setting $z=0$ in (26), and let $\left\{v_{1}, \ldots, v_{n-r}\right\}$ be a basis of $\operatorname{Ker} A$, which can be obtained setting $c=d=0$ and $z=e_{1}, \ldots, e_{n-r}$ in (26), where $\left\{e_{1}, \ldots, e_{n-r}\right\}$ is the standard basis of $\mathbb{R}^{n-r}$. Then we have

$$
\Pi^{\top} x_{L S}=\Pi^{\top} x_{B}-K \lambda
$$

where $K$ is the $n \times(n-r)$ matrix with columns $v_{1}, \ldots, v_{n-r}$ and $\lambda \in \mathbb{R}^{n-r}$ is the least-squares solution of $K \lambda=\Pi^{\top} x_{B}$. Since this last system of equations is over-determined and full-rank, it can be solved by a $\mathrm{QR}$ decomposition without pivoting.

\subsubsection{Parallel strategies}

For the continuation of invariant tori, two parallel strategies have been implemented. In the first one, several families are continued at once, so that at a given moment each processor is working in a different family. In the second one, all the processors work in the same family.

The first strategy is intended for the simultaneous continuation of $n_{c}$ one-parametric families of invariant tori. The "breaking point" to parallelize has been the continuation step. It has been implemented using a master-slave model. The master program spawns $n_{e}$ copies of the slave program and distributes the $n_{c}$ continuations among them. Given a torus of a family, the slave program computes the next by performing one continuation step.

The master program searches for continuation steps to perform cyclically among the families to be continued. In this way, we can carry out simultaneously the continuation of more families than processors $\left(n_{c}>n_{e}\right)$. The slaves are ordered in a list where the master searches for free slaves from the beginning to the end. Then, when more than $n_{c}-n_{e}$ continuations finish and some slaves become idle, the load "goes up" to the beginning of the list of slaves. This allows to take advantage of an heterogeneous network by putting the fastest processors in the beginning of this list.

PVM has been used as message-passing library for the communication between the master and slave processes. For the part of the slave code that solves linear systems, we have used the routines for QR decomposition with and without column pivoting of the LAPACK library [1], mainly DGEQPF, DGEQRF, DORMQR and DTRTRS.

Proceeding in this manner, the maximum values of the parameters reachable are given by the speed and amount of memory available in each node of the parallel computer being used. The second strategy overcomes these restrictions, at the expense of working just in one family.

With respect to this second strategy, it consists on parallelizing every item of section 2.4.2 individually, namely:

- For the evaluation of system (16) and its differential, an strategy is to distribute the $1+$ $2 N_{f}$ numerical integrations to perform among the processors. In our implementation, each process performs just the integrations necessary to generate the piece of matrix it will own during the resolution of linear systems. Depending on the distribution of the matrix, which in our case is given by ScaLAPACK conventions, this can lead to several processes performing the same integrations. An alternative is to distribute the integration in a manner independent of the distribution of the matrix, but this requires extra communication among processes before starting the resolution of linear systems. 
- The resolution of linear systems has been parallelized by using parallel versions of the QR algorithms with and without column pivoting. Our implementation uses the ScaLAPACK library [2], mainly the routines PDGEQPF, PDGEQRF, PDORMQR and PDTRTRS.

- The computation of the bound (17) can be parallelized just by distributing the $M\left(1+2 N_{f}\right)$ numerical integrations to perform among the processors.

As a final remark, note that these two strategies could be combined.

\section{$3 \quad$ Numerical Results}

\subsection{Lyapunov families of periodic orbits around the libration points}

According to Lyapunov's center theorem, there are two families of periodic orbits emanating from each equilibrium point. We will denote these families as the vertical Lyapunov family and the planar Lyapunov family. The starting energy levels for them are those of the corresponding equilibrium points, which are given, for the Earth-Moon mass ratio, in table 1.

\begin{tabular}{|c|c|c|}
\hline$L_{1}$ & $L_{2}$ & $L_{3}$ \\
\hline-1.59417 & -1.58608 & -1.50607 \\
\hline
\end{tabular}

Table 1: Energy values of the three collinear equilibrium points for $\mu=0.012150585$

For all three points, the vertical family starts as an infinitesimal oscillation in the $z$ direction. All three families end at bifurcating planar orbits. For the $L_{1}$ case, the termination orbit surrounds the massive primary $m_{1}$ and the equilibrium points $L_{1}$ and $L_{3}$. This is an orbit of the (g) family, following Stromgren's terminology. For the $L_{2}$ point this planar orbits surrounds both primaries and $L_{1}$ ((l) family), and for $L_{3}$ it surrounds both primaries and the three equilibrium points $((1)$ family). In Fig. 3, we give the characteristic curves (energy vs period) of the three families, as well as their stability curves. When the range of the stability parameters is very large (usually for the $L_{1}$ and $L_{2}$ families), we have plotted $2 \operatorname{arcsinh}($ stab.par.)/ $\operatorname{arcsinh} 2$. In Fig. 4 we show the planar termination orbits of the three families.

Aside from the termination orbit, there appear along the families additional bifurcation orbits. Their energy values are given in table 2 . We will come back to these orbits later on.

\begin{tabular}{|c|r|r|r|}
\hline$\#$ & \multicolumn{1}{|c|}{$L_{1}$} & \multicolumn{1}{c|}{$L_{2}$} & \multicolumn{1}{c|}{$L_{3}$} \\
\hline 1 & -1.49590 & -1.48354 & -0.01537 \\
2 & 0.41391 & 0.55849 & 0.32201 \\
3 & & & 0.54330 \\
\hline
\end{tabular}

Table 2: Energy levels of the bifurcation orbits of the Lyapunov vertical families of periodic orbits. For each $L_{i}$, the last bifurcation orbit corresponds to the termination of the family.

For the planar Lyapunov families around $L_{1}, L_{2}$ and $L_{3}$, which correspond to families (c), (a) and (b) in Stromgren's classification, we have stopped the continuation when a collision orbit 

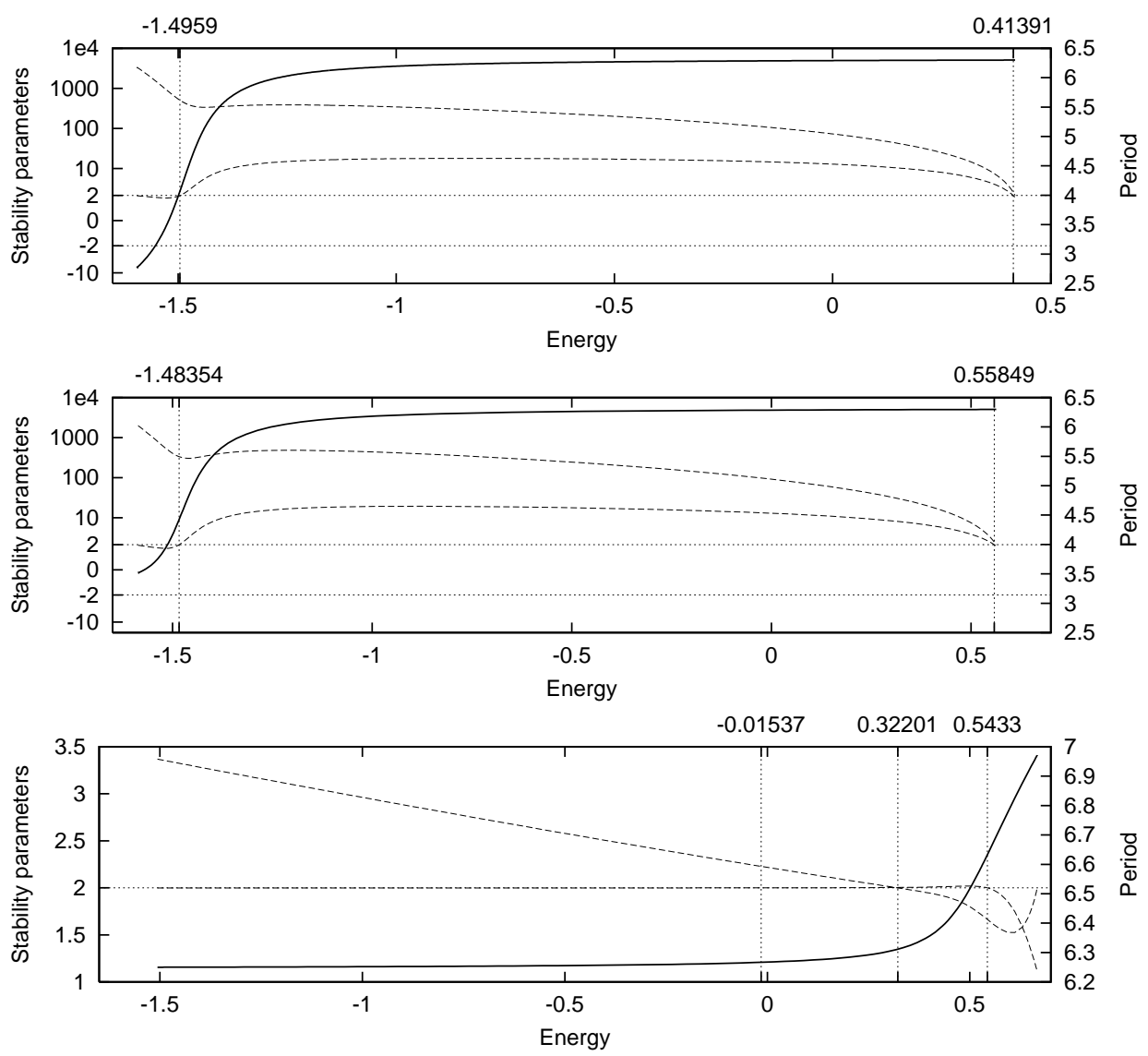

Figure 3: Characteristic curves (solid line) and stability parameters (dashed lines) of the vertical Lyapunov families of periodic orbits for $L_{1}, L_{2}$ and $L_{3}$ (from top to bottom). The vertical lines correspond to the energy levels of the bifurcation orbits (see table 2).

L1

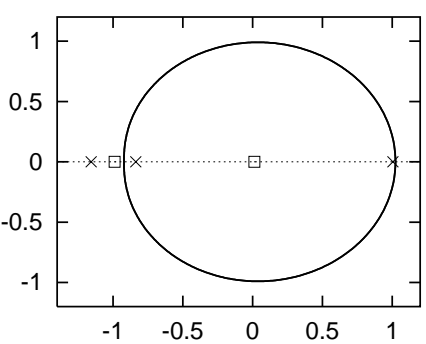

L2

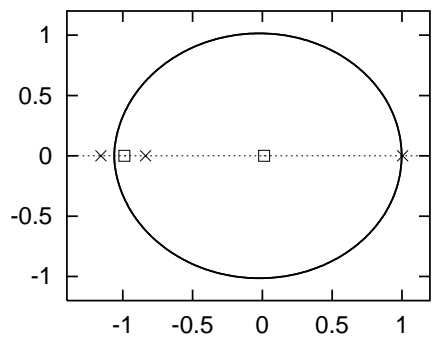

L3

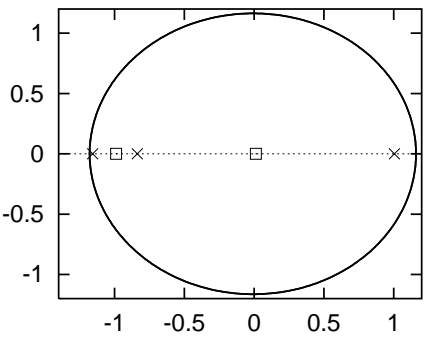

Figure 4: Termination planar orbits of the vertical Lyapunov families of periodic orbits around $L_{1}, L_{2}$ and $L_{3}$. On the $x$ axis we have displayed the position of both primaries ( $\square$ ) and the equilibrium points $(\times)$. The three orbits are traveled clockwise. 
with one of the primaries appears. This is due to the fact that we are only interested in the dynamics in a neighborhood of the libration points, and the collision orbits are already large (see Fig.6). For $L_{1}$ and $L_{3}$ the collision takes place with the largest primary, whereas for the $L_{2}$ case it happens with the small one. In Fig. 5 we give the characteristic and stability curves for these families.
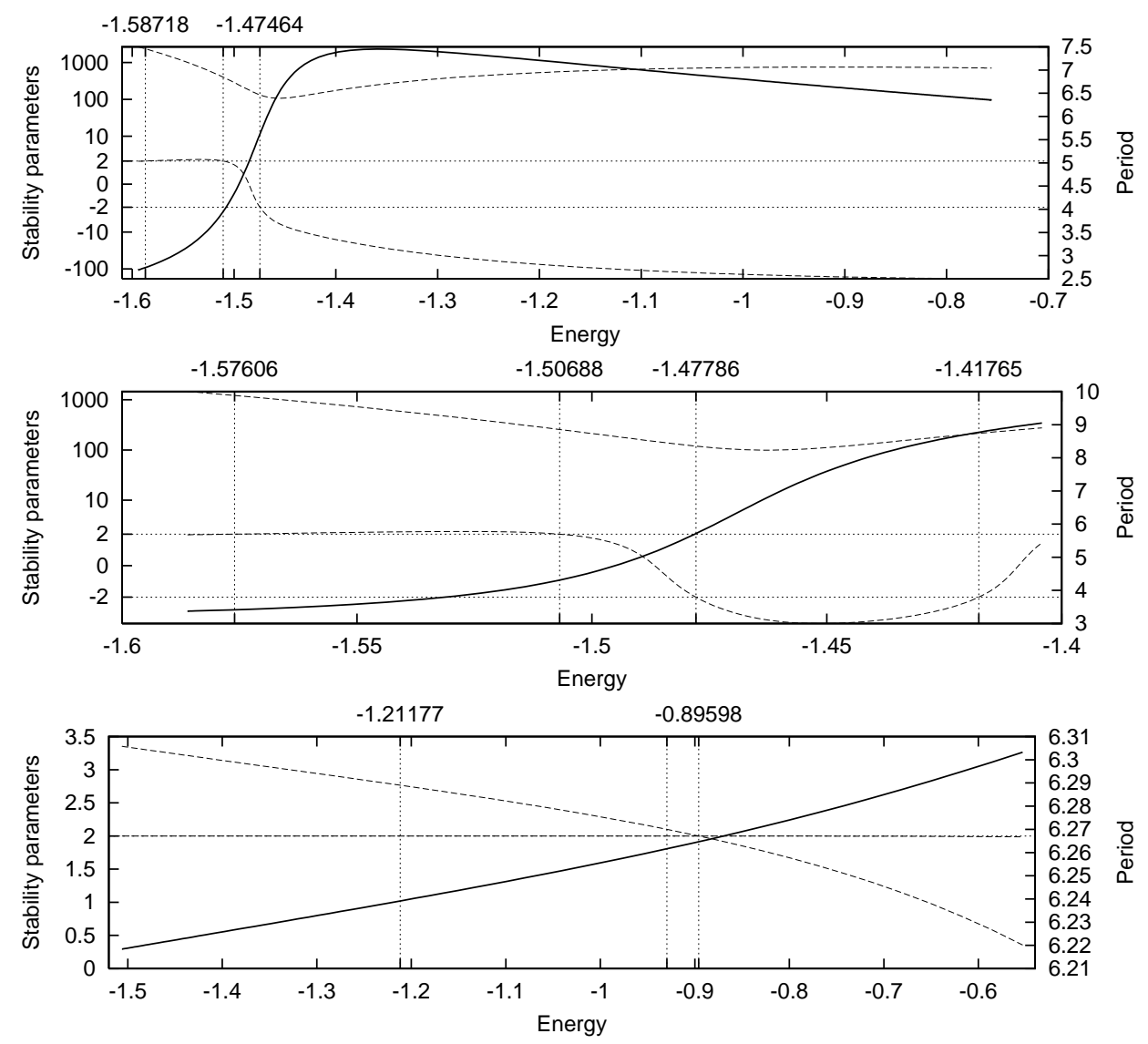

Figure 5: Characteristic curves (solid line) and stability parameters (dashed lines) of the planar Lyapunov families of periodic orbits for $L_{1}, L_{2}$ and $L_{3}$ (from top to bottom). The vertical lines correspond to the energy levels of the bifurcation orbits (see table 3 ).

According to Hénon ([10]), the vertical stability character of a planar periodic orbit indicates whether or not this orbit is at the same time a member of a family of 3D periodic orbits. Only what Hénon calls vertical critical orbits $\left(\left|a_{v}\right|=1\right)$ can be embedded in 3D families of periodic orbits. Considering small out-of-plane perturbations of the periodic orbit after one revolution, and using

$$
\left(\begin{array}{c}
\Delta z \\
\Delta \dot{z}
\end{array}\right)_{\text {final }}=\left(\begin{array}{ll}
a_{v} & b_{v} \\
c_{v} & d_{v}
\end{array}\right)\left(\begin{array}{c}
\Delta z \\
\Delta \dot{z}
\end{array}\right)_{\text {initial }},
$$

three different kinds of bifurcated solutions can be considered (see [10]):

- Type A: Symmetrical periodic orbits with respect to the $x y$-plane and generated from planar orbits with $a_{v}=1, c_{v}=0$. 
L1

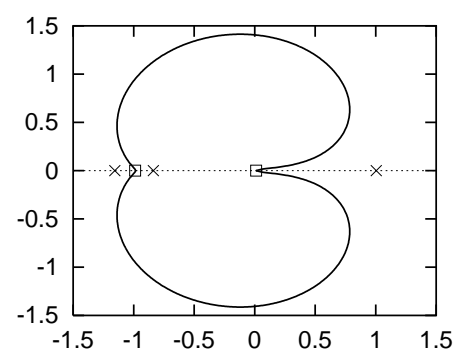

L2

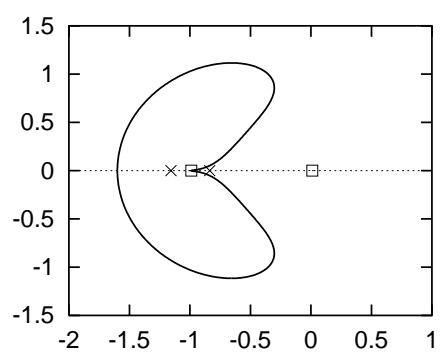

L3

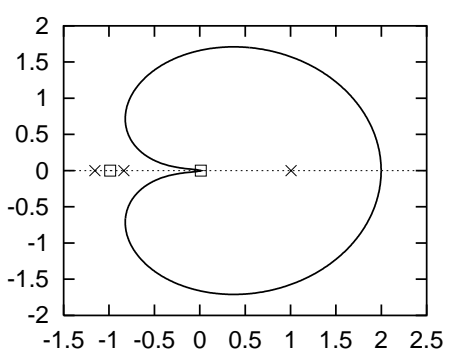

Figure 6: First collision orbits of the Lyapunov families of periodic planar orbits around $L_{1}, L_{2}$ and $L_{3}$. On the $x$ axis we have displayed the position of both primaries $(\square)$ and the equilibrium points $(\times)$. The three orbits are traveled clockwise.

- Type B: Symmetrical periodic orbits with respect to the $x$-axis, generated from planar ones with $a_{v}=1, b_{v}=0$.

- Type C: Symmetrical periodic orbits with respect to both the $x y$-plane and the $x$-axis, generated from planar periodic orbits with $a_{v}=-1$ and $c_{v}=0$ or $a_{v}=-1$ and $b_{v}=0$.

In table 3 we give the bifurcations of the three families before the appearance of a collision orbit. Further bifurcations can be found in [10] for the value of the mass ratio $\mu=0.5$. The bifurcated families will be described in the following sections. In all three cases, the first bifurcated family is formed by the so-called halo type orbits. These are 3D periodic orbits symmetric with respect to the $\{y=0\}$ plane.

For the three equilibrium points, the two Lyapunov families are connected by two-lane bridges of periodic orbits going from bifurcation orbit \#1 of the vertical family to bifurcation orbit \#2 (B type) of the planar one. Each orbit in one lane has a symmetrical orbit, with respect to the $\{z=0\}$ plane, in the other lane. In Fig. 7 the characteristic and stability curves for these families are displayed. Just for illustrating purposes, we show some orbits of the bridge in the $L_{1}$ case in Fig. 8.

\subsection{Halo-type orbits}

As it has already been said, from the three planar Lyapunov families bifurcate families of 3D periodic orbits which are symmetrical with respect to the $\{y=0\}$ plane. These orbits are known as halo orbits. There are other orbits with the same symmetry (and a similar shape) which bifurcate from the families of halo orbits by duplication, triplication, etc., of the period. This section is devoted to these kind of families.

At each planar bifurcation orbit number 1 (table 3) start two symmetrical families of periodic orbits with respect to the $\{z=0\}$ plane. They are known as north and south class halo families, according to the maximum value of $|z|$ along the orbit. The bifurcation orbits at which they are born correspond to a 1:1 resonance between the (planar) proper and one of the (vertical) normal frequencies. These orbits are well-known in the literature ([20]).

Their characteristic and stability curves are displayed in Fig. 9. The north and south class families around $L_{1}$ end simultaneously at a plane orbit around both primaries and $L_{1}, L_{2}$, which 
L1
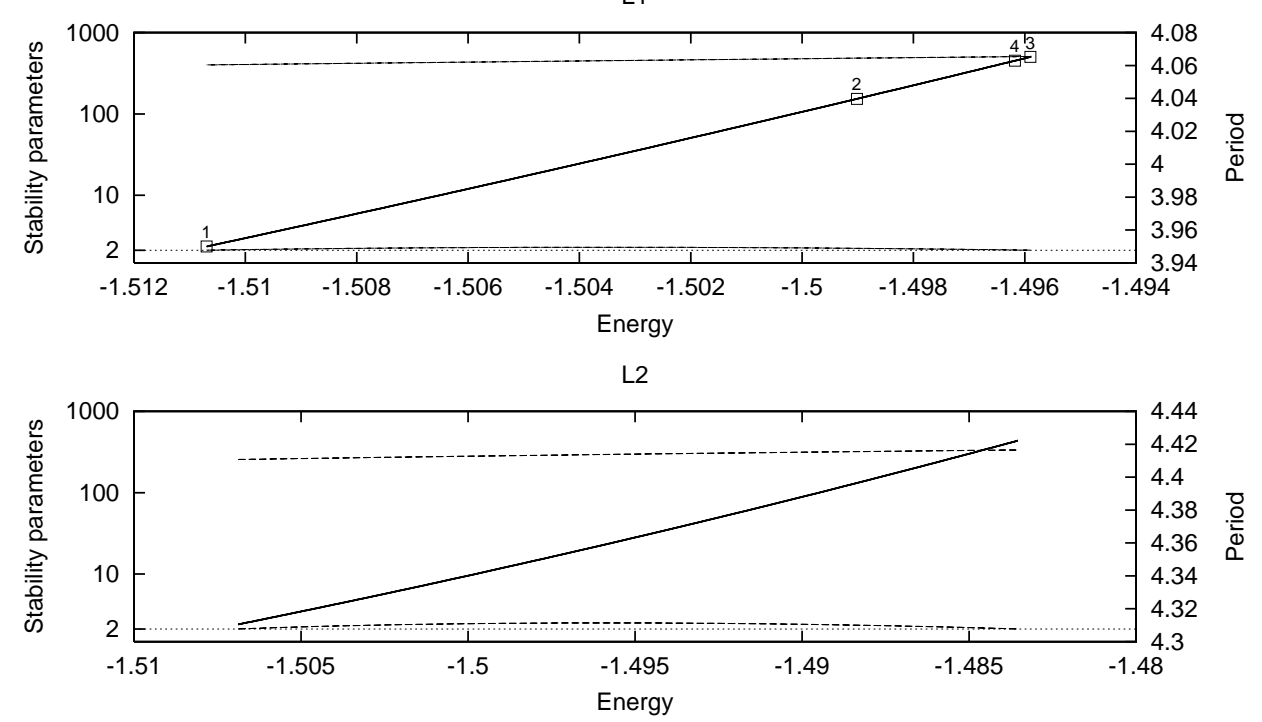

L3

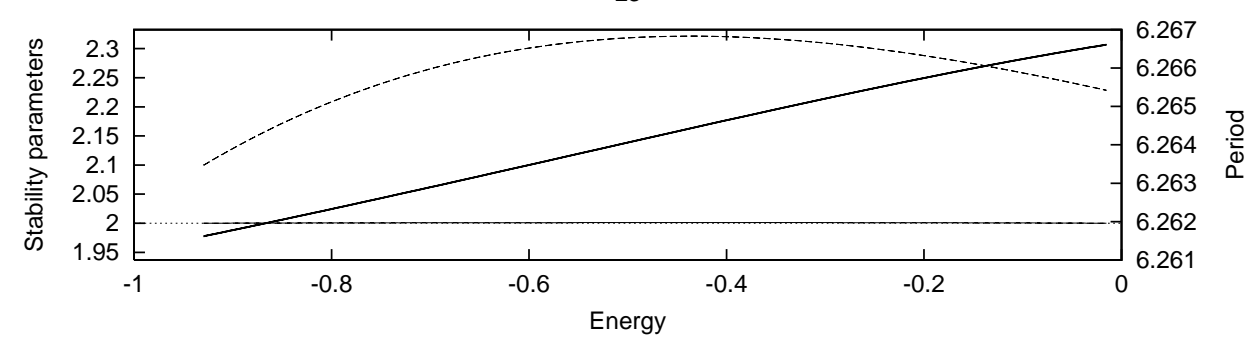

Figure 7: Characteristic curves (solid line) and stability parameters (dashed lines) of the bridges between the planar Lyapunov families and the vertical ones for $L_{1}, L_{2}$ and $L_{3}$.

\begin{tabular}{|c|cc|cc|cc|}
\hline & \multicolumn{2}{|c|}{$L_{1}$} & \multicolumn{2}{c|}{$L_{2}$} & \multicolumn{2}{c|}{$L_{3}$} \\
$\#$ & Energy & Type & Energy & Type & Energy & Type \\
\hline 1 & -1.58718 & $\mathrm{~A}$ & -1.57606 & $\mathrm{~A}$ & -1.21177 & $\mathrm{~A}$ \\
2 & -1.51070 & $\mathrm{~B}$ & -1.50688 & $\mathrm{~B}$ & -0.92954 & $\mathrm{~B}$ \\
3 & -1.47464 & $\mathrm{C}$ & -1.47786 & $\mathrm{C}$ & -0.89598 & $\mathrm{~B}$ \\
4 & & & -1.41765 & $\mathrm{C}$ & & \\
\hline
\end{tabular}

Table 3: Energy levels and type of the vertical critical orbits of the Lyapunov planar families of periodic orbits. 
Planar orbit

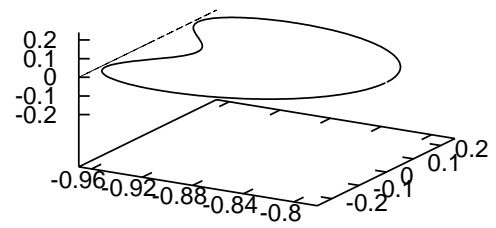

Orbit 1, lane 1

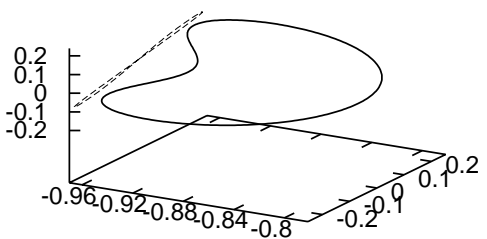

Orbit 2, lane 1

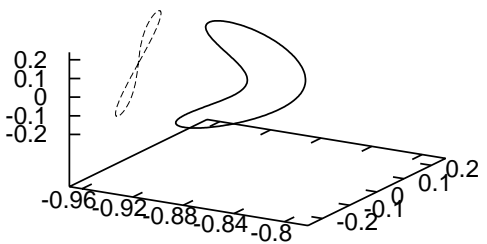

Orbit 1, lane 2

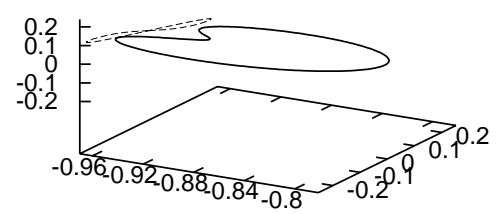

Orbit 2, lane 2

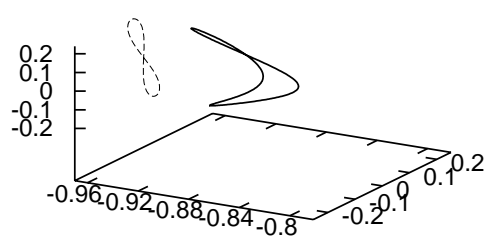

Vertical orbit

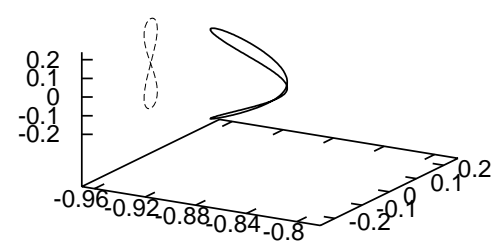

Figure 8: 3D projections (solid lines) and $y z$ projections (dashed lines) of some periodic orbits of the bridge connecting the two Lyapunov families in the $L_{1}$ case. The orbit in the top is the planar bifurcation orbit, and the one in the bottom is the vertical bifurcation one. The orbits at both sides are on the two lanes of the bridge connecting the two previous orbits. 
is of family (1) in Stromgren's classification. For the other two equilibrium points, we have not followed the families up to their natural termination and the computations have been stopped when a collision orbit has been detected.

A comment is necessary about the representation of the stability parameters. In the $L_{1}$ case there appears a bifurcation to complex instability. This means that two pairs of elliptic eigenvalues moving in the unit circle "collide", and after the collision leave the unit circle. In this way, the stability parameters become complex conjugate (they are real in both the hyperbolic and the elliptic case). This happens at energies -1.47034 and 0.47747 . In this situation we have represented the real and imaginary parts of one of the stability parameters, as can be better appreciated in Fig. 10, which is a magnification of the $L_{1}$ case in Fig. 9.
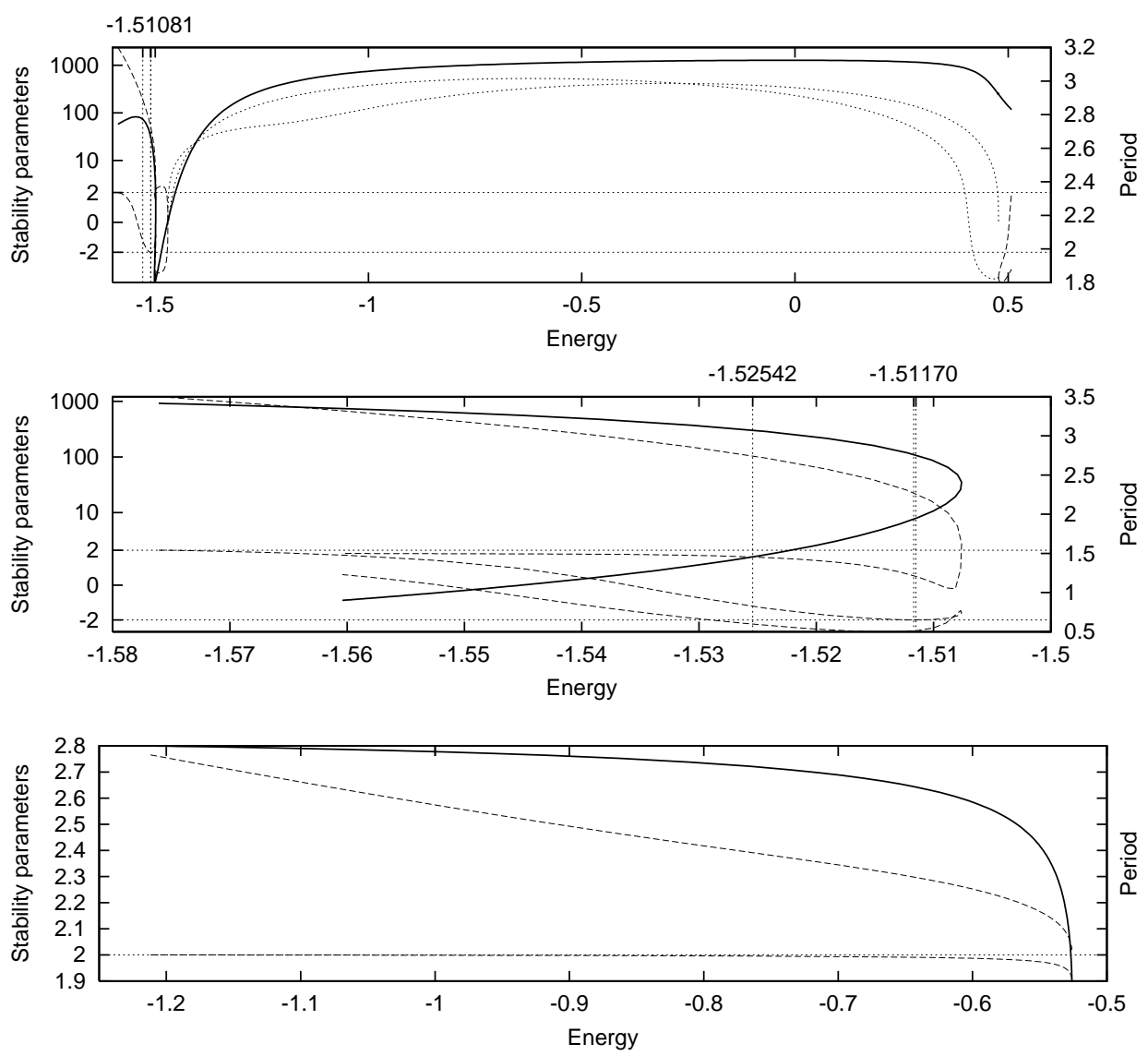

Figure 9: Characteristic curves (solid line) and stability parameters (non-solid lines) of the halo families around $L_{1}, L_{2}$ and $L_{3}$ (from top to bottom). For the $L_{1}$ case, we have represented with dashed lines the stability parameters when they are real, and with dotted lines the real and imaginary parts of one of them in the complex case. The vertical lines correspond to the energy levels of the bifurcation orbits (see table 2).

As in the Lyapunov families, we have computed some bifurcation orbits, whose energy values are displayed in table 4 . The first two bifurcations are period doubling, since they correspond to a value of the small stability parameter equal to $2 \cos (2 \pi / 2)=-2$. At the first bifurcation the bifurcated family has one elliptic stability parameter, while the second bifurcation has both 

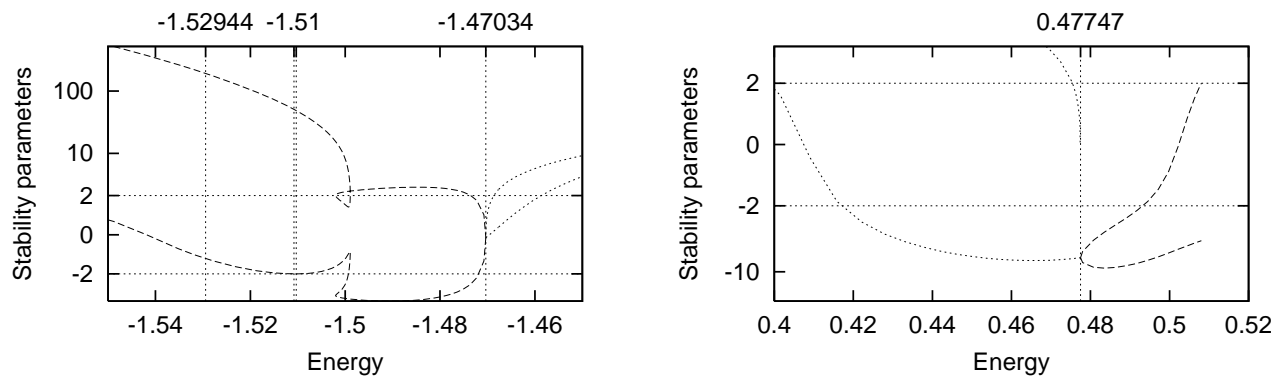

Figure 10: Magnification of the $L_{1}$ stability curves near the bifurcations to complex instability. The vertical lines correspond to the energy levels of the bifurcation orbits of table 2 and the complex instability bifurcations (see explanations in the text).

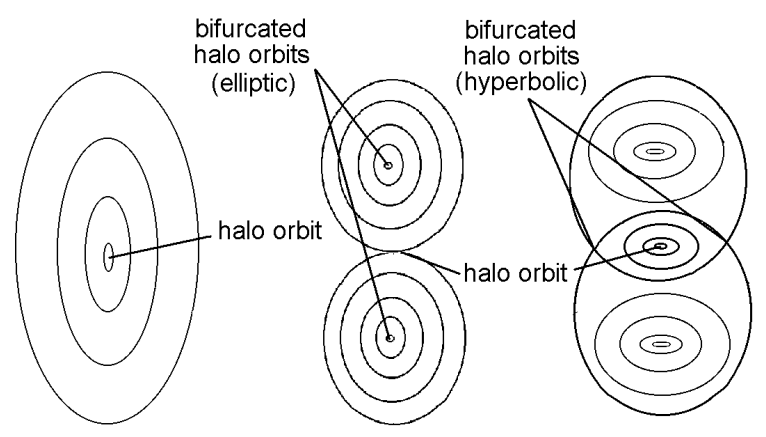

Figure 11: Qualitative representation of the two period-doubling bifurcations of the $L_{1}$ halo family.

stability parameters hyperbolic. These two bifurcations are represented qualitatively in figure 11. As for the third bifurcation orbit, the small stability parameter equals to $2 \cos (2 \pi / 3)$, so it gives rise to two period triplicated families. One of the bifurcated families has an elliptic stability parameter, whereas the other has both stability parameters hyperbolic. We must remark that, for the range of the halo family around $L_{3}$ explored, there are no bifurcations due to low-order resonances. In a next section we will show the role played by these bifurcations in the geometry of the phase space.

\begin{tabular}{|c|c|c|}
\hline$\#$ & $L_{1}$ & $L_{2}$ \\
\hline 1 & -1.51081 & -1.51170 \\
2 & -1.51033 & -1.51150 \\
3 & -1.52944 & -1.52542 \\
\hline
\end{tabular}

Table 4: Energy values at the period doubling and triplication bifurcation orbits of the halo families (see the details in the text).

The characteristic curves for these families are given in figures 12 and 13. A comment must be made on the criteria used to stop the continuation of these families. In some cases we have reached a natural termination at a bifurcation orbit. When this has not been possible, we have 
used as stopping criteria either

- the detection of a collision orbit inside the family, or

- reaching high periods (over 40 RTBP time units).

Table 5 gives the contingency that produced the stopping of each continuation.
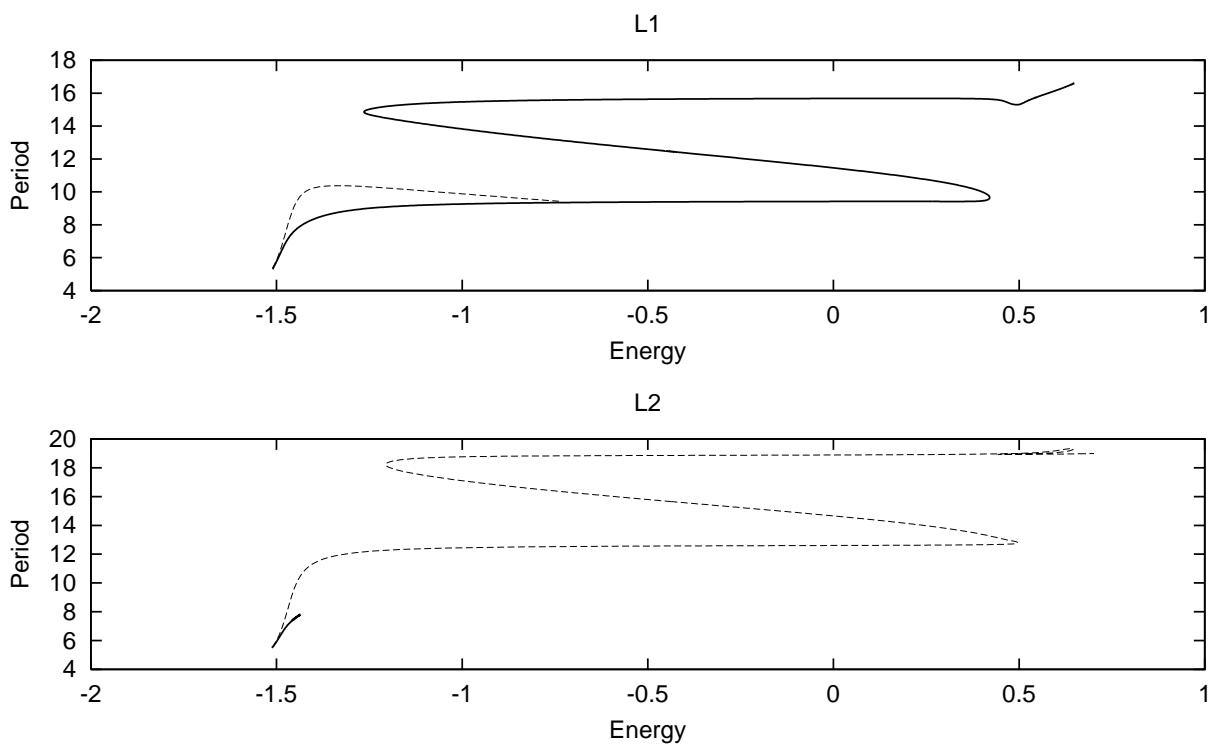

Figure 12: Characteristic curves of the families that bifurcate by period duplication from the halo families of $L_{1}$ and $L_{2}$. The solid lines represent the hyperbolic families, and the dashed ones the elliptic.

\begin{tabular}{|c|c|c|c|c|}
\hline \multirow{2}{*}{} & \multicolumn{2}{|c|}{$L_{1}$} & \multicolumn{2}{c|}{$L_{2}$} \\
\cline { 2 - 5 } & hyperbolic & elliptic & hyperbolic & elliptic \\
\hline period duplication & natural term. & $m_{1}$ collision & $m_{2}$ collision & high period \\
\hline period triplication & high period & $m_{1}$ collision & $m_{2}$ collision & high period \\
\hline
\end{tabular}

Table 5: Ending conditions of the continuations of the families bifurcated by period duplication and triplication from the halo families of $L_{1}$ and $L_{2}$.

In order to illustrate how orbits in these families "unfold" from a halo orbit, we give in figures 14 and 15 some orbits of the elliptic bifurcation by period duplication and of the hyperbolic bifurcation by period triplication, respectively. Both families correspond to the $L_{1}$ point.

\subsection{Families of invariant tori}

As a first example of the methodology described for the computation of families of tori, we will show the results related to the computation of a family of tori that starts at a vertical Lyapunov 
L1
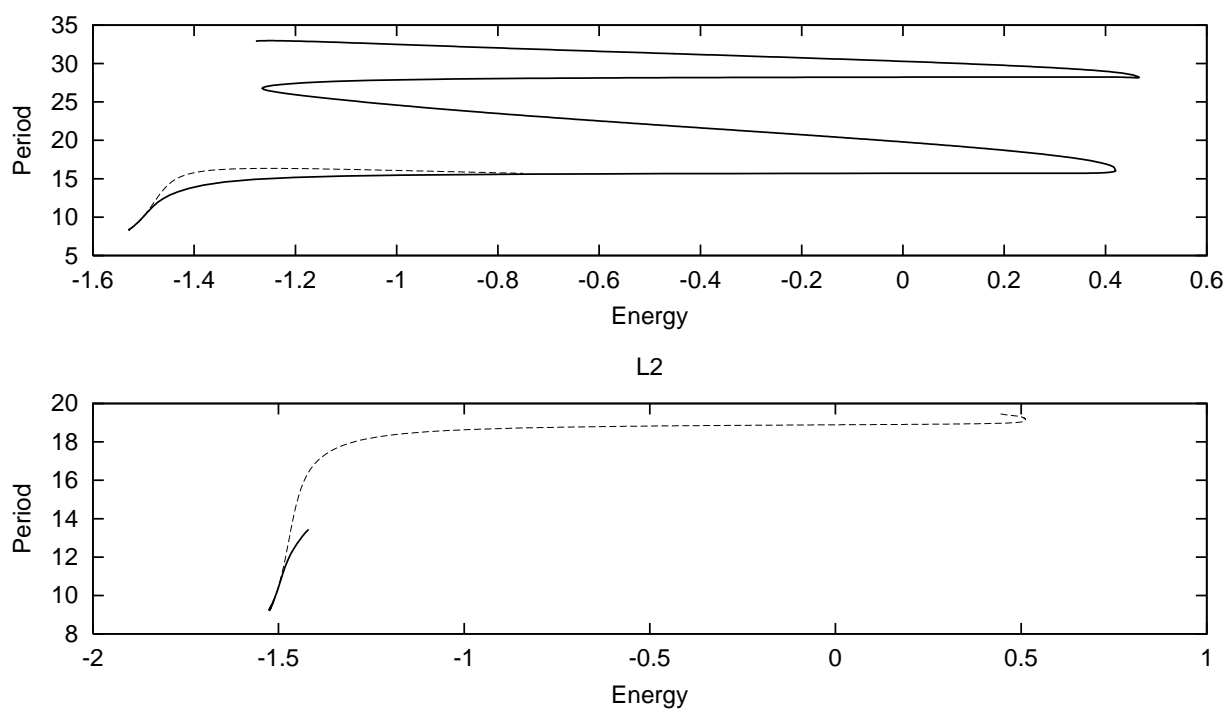

Figure 13: Characteristic curves of the families that bifurcate by period triplication from the halo families of $L_{1}$ and $L_{2}$. The solid lines represent the hyperbolic families, and the dashed ones the elliptic.

orbit around $L_{1}$ at the energy level -1.59 . We will follow this family keeping fixed the value of the energy. For this value of the energy, the family ends at a planar Lyapunov orbit.

To start the computation of the family, an initial torus was estimated starting longitudinally to the vertical orbit. In a first step, this torus was refined keeping constant $h, A_{0}^{z}$ (to fix the first invariant curve on the torus), $A_{1}^{x}$ (to avoid falling back to the p.o.) and $A_{1}^{y}$ (to avoid coefficient modulation). In the next steps of the predictor-corrector scheme, we have kept $h$ and $A_{0}^{z}$ fixed for the predictor (in order to have a 2D kernel to choose a direction orthogonal to coefficient modulation, as has been already described), and for the corrector we have added a new coordinate chosen according to the procedure described in section 2.2.1. We have used a multiple shooting strategy with two invariant curves $(m=2)$. All the Fourier coefficients just mentioned correspond to the first one.

At the beginning, $\delta$ is taken as the period of the vertical orbit and the rotation number $\rho$ is related to a normal frequency of the starting vertical orbit. More concretely, $2 \cos \rho$ is the smallest stability parameter of the vertical orbit. At the end of the continuation, we get two new values of the parameters $\rho$ and $\delta$. Now $\rho$ is related to the frequency of the ending planar orbit through

$$
\rho=\delta \frac{2 \pi}{T}-2 \pi
$$

where $T$ is the period of the ending planar orbit. The final value of the parameter $\delta$ is checked to be

$$
\delta=\frac{2 \pi}{2 \pi-\nu} T
$$

where $2 \cos \nu$ is the smallest stability parameter of the planar orbit. This determines the sign of $\nu$ and the value of $j$ in equations (24) and (25). These values will be used in the next section when performing the constant rotation number continuations starting from the planar orbits. 
Orbit 1, 3D view.

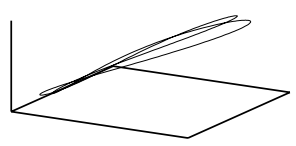

Orbit 1, xz projection.

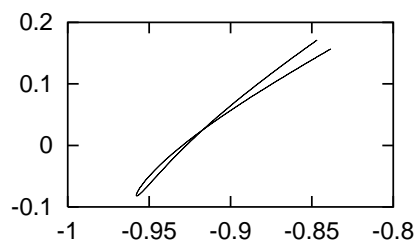

Orbit 2, 3D view.

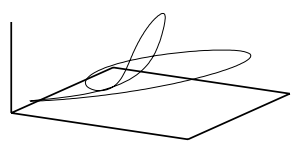

Orbit 2, xz projection

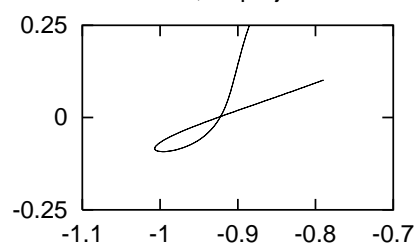

Orbit 3, 3D view.

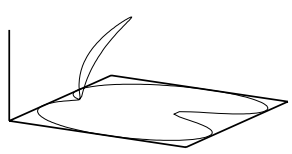

Orbit 3, xz projection.

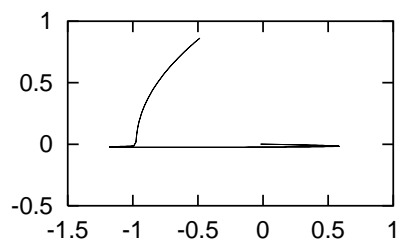

Orbit 1 , xy projection.

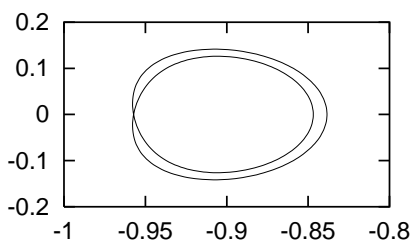

Orbit 1, yz projection.

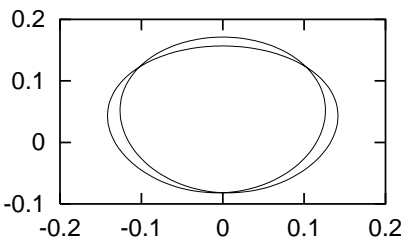

Orbit 2, xy projection.

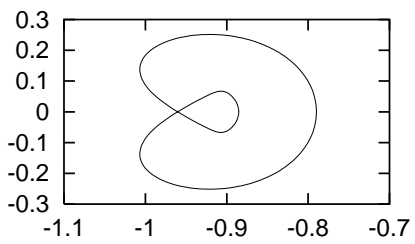

Orbit 2, yz projection.

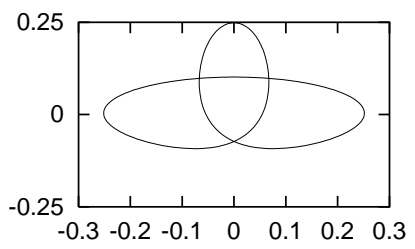

Orbit 3, xy projection.

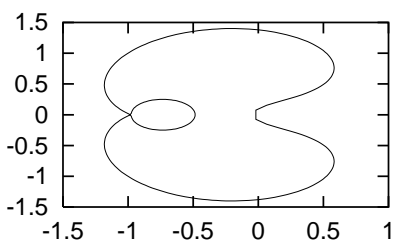

Orbit 3, yz projection.

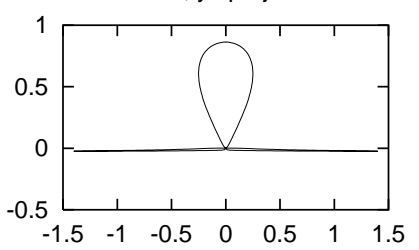

Figure 14: Some orbits of the elliptic bifurcation by period duplication of the $L_{1}$ halo family. Orbit 1: energy $=-1.51061$, period $=5.34666$. Orbit 2: energy $=-1.49158$, period $=6.35119$. Orbit 3: energy $=-$ 0.99683 , period $=9.87531$. 
Orbit 1, 3D view.

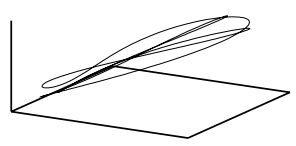

Orbit $1, x z$ projection

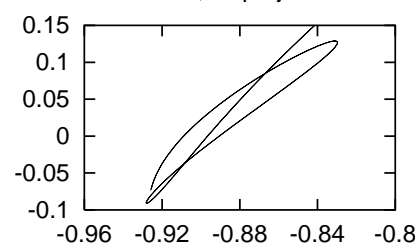

Orbit 2, 3D view.

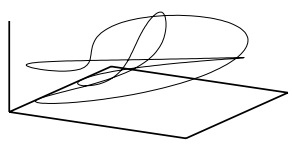

Orbit 2, xz projection

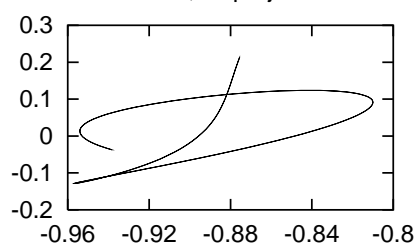

Orbit 3, 3D view.

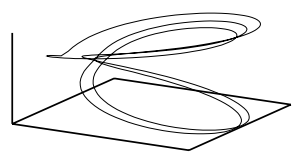

Orbit 3, xz projection.

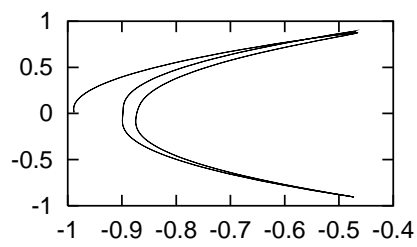

Orbit 1 , xy projection.

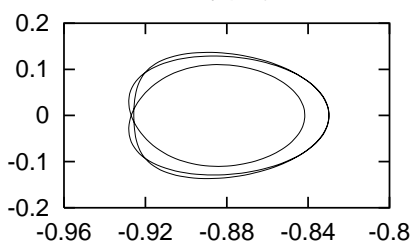

Orbit 1, yz projection.

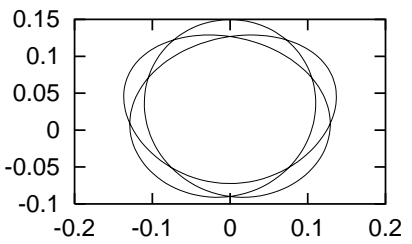

Orbit 2, xy projection.

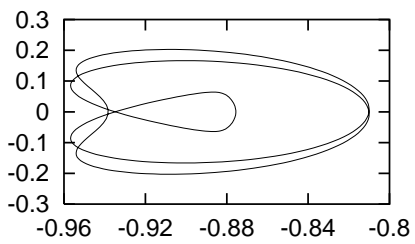

Orbit 2, yz projection.

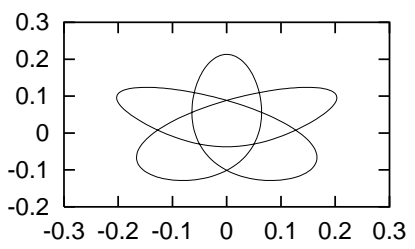

Orbit 3, xy projection.

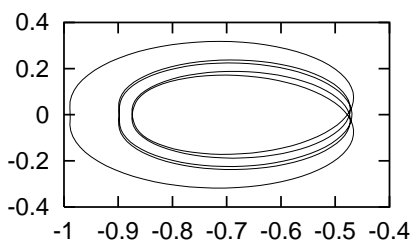

Orbit 3, yz projection.

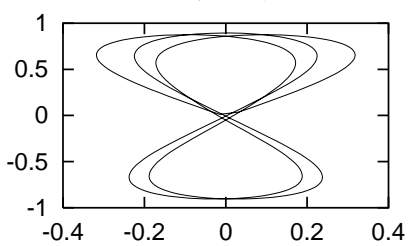

Figure 15: Some orbits of the hyperbolic bifurcation by period triplication of the $L_{1}$ halo family. Orbit 1: energy $=-1.52853$, period $=8.36012$. Orbit 2: energy $=-1.51026$, period $=9.36247$. Orbit 3: energy $=-0.99208$, period $=15.46609$. 
Some tori of this continuation are shown in Fig. 16.

The next step is to extend the above computations to energy values covering the full range of vertical Lyapunov periodic orbits with central part.

\subsubsection{Invariant tori starting around vertical orbits}

In Fig. 17 we have displayed, for the three equilibrium points, the regions, in the energy-rotation number plane, covered by the 2-parametric family of tori computed starting from the vertical Lyapunov families of periodic orbits. Each region is surrounded by a curve formed by several pieces:

- The lower left piece $\alpha$ (from vertex 1 to 2 ) is related to the the planar Lyapunov family. The orbits of this family represented in the curve are just the first piece of the family with central part. The horizontal coordinate is the energy level $h$ of the curve and the vertical coordinate is $\rho=\frac{(2 \pi)^{2}}{2 \pi-\nu}-2 \pi$, where $2 \cos \nu$ is the stability parameter of the orbit.

- The upper piece $\beta$ (from vertex 2 to 3 ) is strictly related to the vertical Lyapunov family. The points on this curve are $(h, \rho)$ where $h$ is the energy of the orbit and the rotation number $\rho$ is such that the elliptic stability parameter of this orbit is $2 \cos \rho$. Note that this relation between $\rho$ and $\nu$ is different from the previous item. This is because, in order to have continuity of $\rho$ along an isoenergetic family of tori, we are forced to begin/end transversally at one family (the planar one, in our case) and end/begin longitudinally at the other (vertical), or vice-versa.

- The bottom boundary $\gamma$ (from vertex 3 to 1 ), that corresponds to $\rho=0$, begins at the value of the energy where the halo families are born. It is related to a separatrix between the tori around the vertical Lyapunov families and the halo ones.

In the three diagrams, there is a narrow range of energies (in the left-hand side of the plots) for which the isoenergetic families of tori start at a vertical p.o. and end at a planar orbit, as was the case in the example of the beginning of this section.

There are different ways for computing the tori within the region surrounded by the curves mentioned above. We always start from the pieces of boundary formed by periodic orbits. One possibility is then to perform the continuation procedure keeping fixed the value of the energy $h$. Another one is to allow variation of the energy but keeping fixed the rotation number $\rho$. In this last case, and in order to be as close as possible of conditions that guarantee the existence of tori for the nonlinear system, it is convenient to set the rotation number "as irrational as possible". To this end, when we have used this second strategy, we have set the values of $\rho$ such that $\frac{2 \pi}{\rho}$ is an integer plus the golden number $\left(\frac{1+\sqrt{5}}{2}\right)$. In both cases, and for all $L_{1}, L_{2}$ and $L_{3}$ cases, we have always reached a region where the number of harmonics $N_{f}$ is larger than the maximum value allowed, which at most has been set equal to 100 . Larger values of this parameter make computing time prohibitive. Just to have an idea of the computing effort, the constant rotation number family with $\rho=0.176$ requires about 3 days of CPU time of an Intel Pentium III at $500 \mathrm{MHz}$, and the complete picture around 100 days (this means around 1.5 days in our Beowulf cluster).

For $L_{1}$ and $L_{2}$, the continuations have been done keeping constant the rotation number, starting longitudinally from the vertical p.o. ( $\beta$ curve) and transversally from the planar p.o. $(\alpha$ 

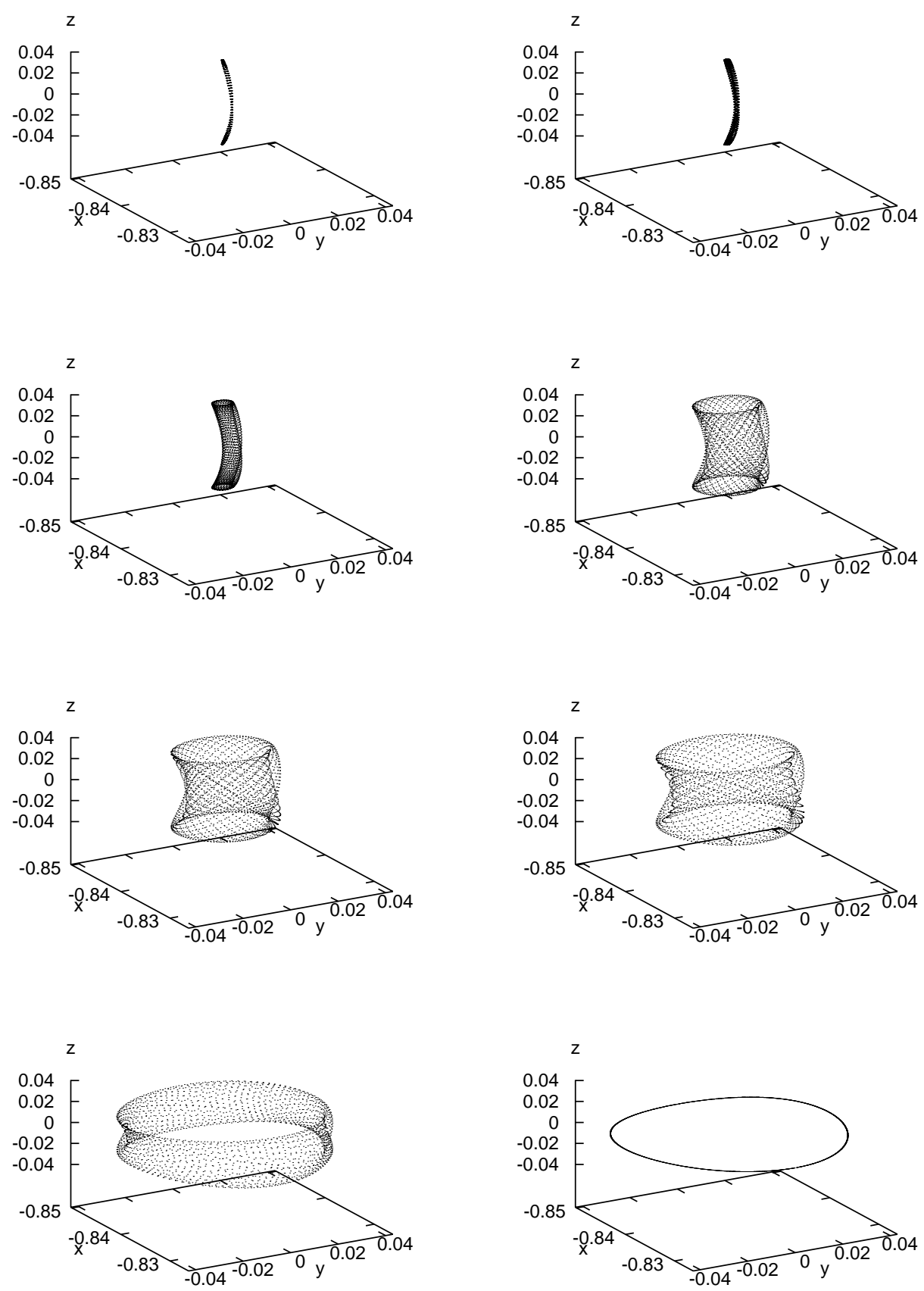

Figure 16: Isoenergetic family of 2D tori starting at a vertical Lyapunov orbit and ending at a planar one. Energy level: -1.59 , equilibrium point: $L_{1}$. 
L1

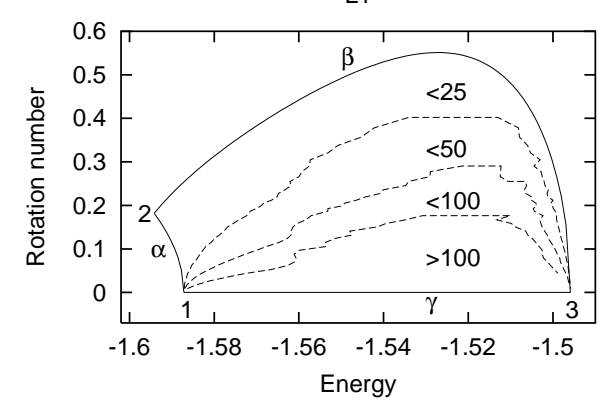

L2

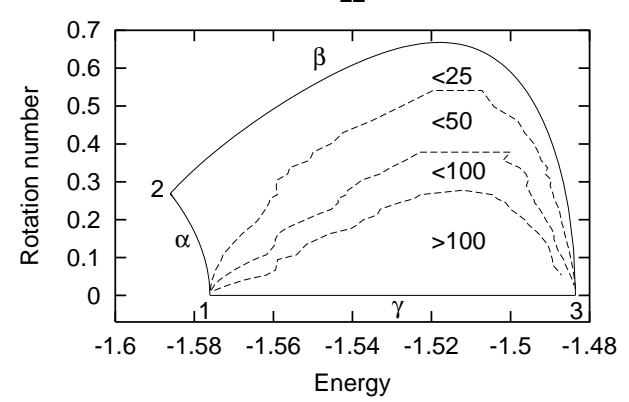

L3

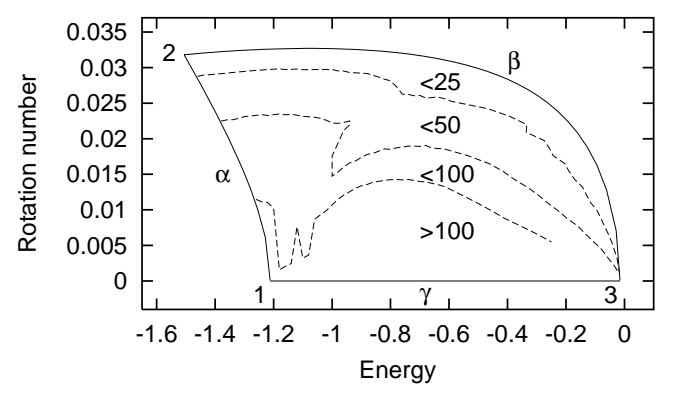

Figure 17: Regions in the energy-rotation number plane covered by the two-parametric family of tori computed starting at the vertical Lyapunov families of p.o. for $L_{1}, L_{2}$ and $L_{3}$. The number of harmonics used for the computation of the tori $(<25,<50,<100$ and $>100)$ is shown in the figures. Vertex 1 is at the value of the energy at which the halo families is born. Vertex 2 is at the value of the energy of the equilibrium point. Vertex 3 is at the value of the energy of the first bifurcation of the vertical Lyapunov family (see tables 3,1 and 2, respectively). 
curve). When starting transversally, the sign of $\nu$ and the value of $j$ of equations (24) and (25) have been adjusted as it has been explained at the beginning of this section. The computations for $L_{3}$ have been done starting (longitudinally) from vertical orbits only, but keeping constant the value of the energy.

\subsubsection{Invariant tori starting around halo and halo-type orbits}

In this section we will give a brief summary about the results obtained relative to tori around halo orbits, as well as the bifurcated families obtained by duplication and triplication of the period.

For the exploration corresponding to the $L_{1}$ case, we have used the piece of the halo family that starts at the bifurcation from the planar family, at energy -1.58718 , and ends at the local maximum of the small stability parameter at energy -1.49899. This is the maximum energy value for which the halo family of p.o. has central part (see Fig. 10). For $L_{2}$, the initial value of the energy is -1.57606 and the value of the energy at the local maximum is -1.50769 (see Fig. 9). The range of energy values explored for $L_{3}$ begins at -1.21177 and ends when a close approach to the larger primary appears.

As in the computations in the previous section, we have used both kinds of continuations (keeping fixed either $h$ or $\rho$ ) for these explorations. Also as before, all the computations have been stopped when the maximum number of harmonics allowed (100) is reached. In Fig. 18 we display the regions in the $h, \rho$ plane where these tori do exist. In the same figures, the number of harmonics needed in order to have a tolerance less than $10^{-10} \mathrm{in}(17)$ is given. All the computations have been done using at most $m=4$ for the multiple shooting procedure.
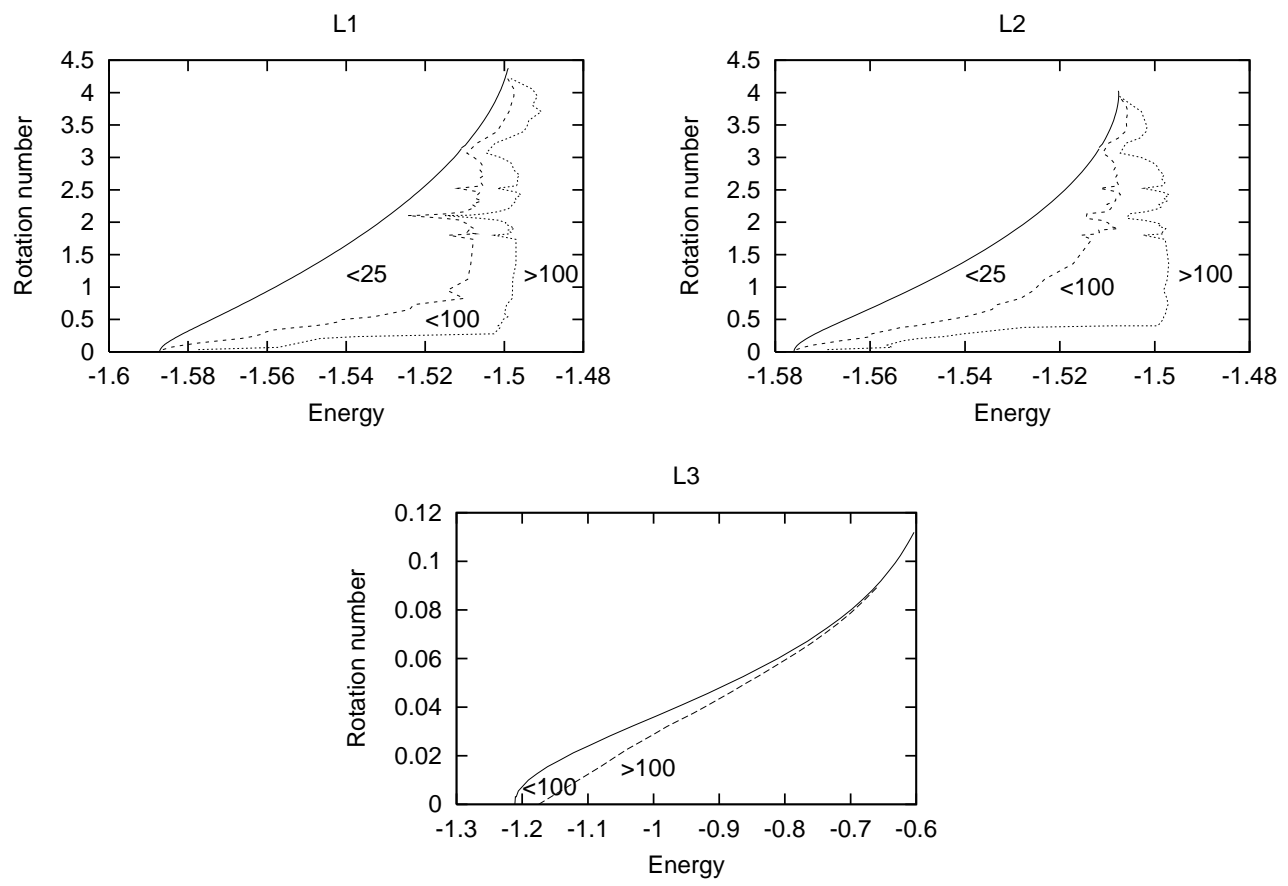

Figure 18: Regions in the energy-rotation number plane covered by the two-parametric family of invariant tori around the halo families around $L_{1}, L_{2}$ and $L_{3}$. As in the previous figure, we show the number of harmonics used for the computation of the tori. 
The bottom border of these regions is shared with the ones displayed in Fig. 17. The lowerleft vertex of the three plots correspond to vertex number 1 of the plots in Fig. 17. This vertex corresponds to the bifurcating planar orbit that gives rise to the two halo families. In all the cases, as $\rho \rightarrow 0$, we are approaching to a (transversal) homoclinic connection [8].

The right-hand side border is in some sense fictitious since it corresponds to reaching the maximum number of harmonics allowed. Anyway, it reflects in a clear way the crossings of the rotation number $\rho$ through low-order resonances, at which the tori collapse to periodic orbits. The four main peaks detected correspond to values of the rotation number equal to $2 \pi / 2,4 \pi / 5,2 \pi / 3$, $2 \pi / 4$ (from top to bottom). The first and third peaks correspond to the 1:3 and 1:2 resonances (the 1:1 resonance, $\rho=0$, is related to the origin of the halo family) related to the bifurcating families of halo-type orbits already mentioned. As it was discussed, some of these families have central part, so we can expect to find invariant tori around them. They have been also computed, and the $h-\rho$ diagrams are given in figures 19 and 20 .
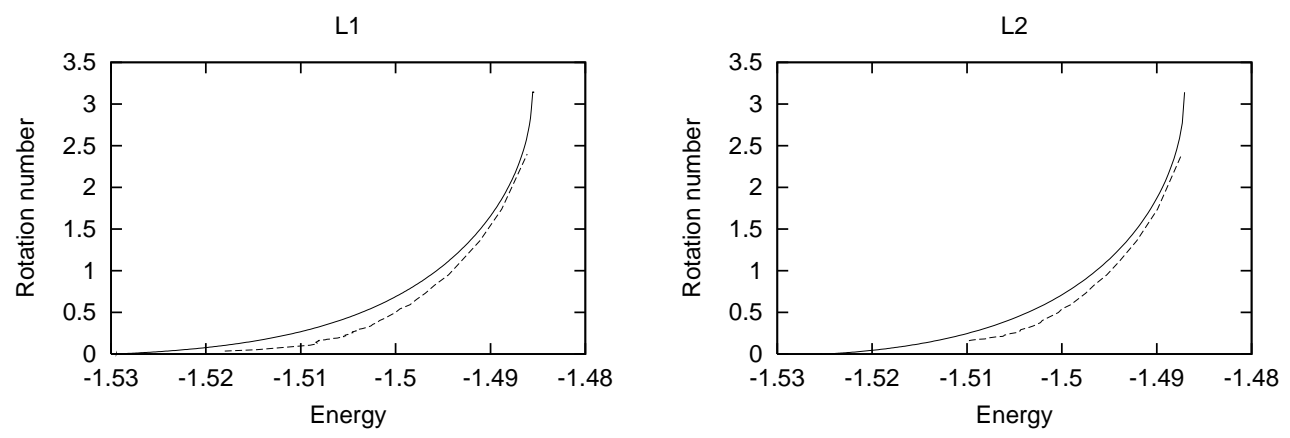

Figure 19: Regions in the energy-rotation number plane covered by the two-parametric families of invariant tori around the elliptic families bifurcated from halo orbits by period triplication. Between the solid curve and the dotted one, the number of harmonics required for the computation of tori is less than 100 . To the right of the dotted curve more than 100 harmonics are required.
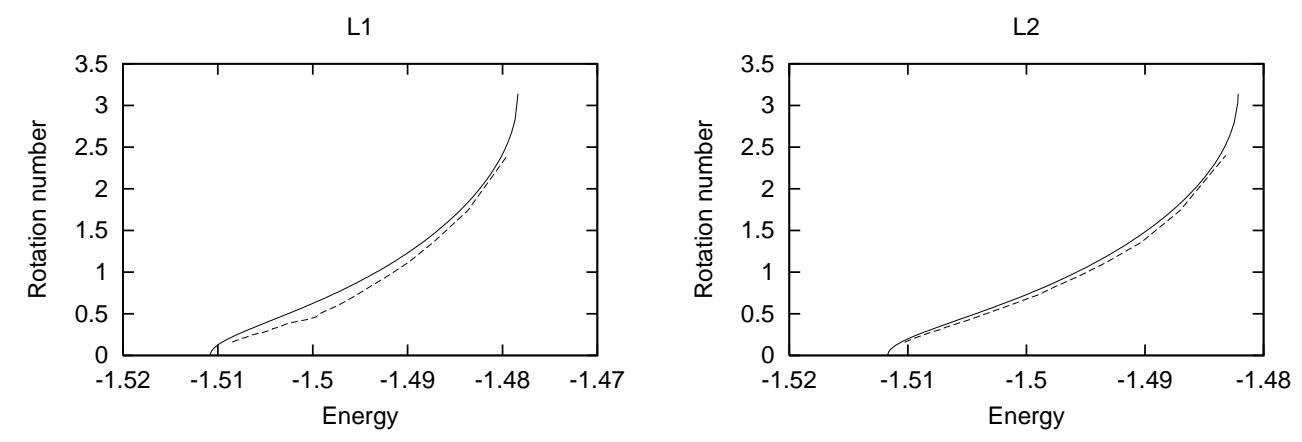

Figure 20: Regions in the energy-rotation number plane covered by the two-parametric families of invariant tori around the elliptic families bifurcated from halo orbits by period duplication. The meaning of the dotted line is the same as in figure 19.

Just for illustrating purposes, in Fig. 21 we have displayed some of the tori that appear around 
the families bifurcated from halo orbits.

L1, period duplication

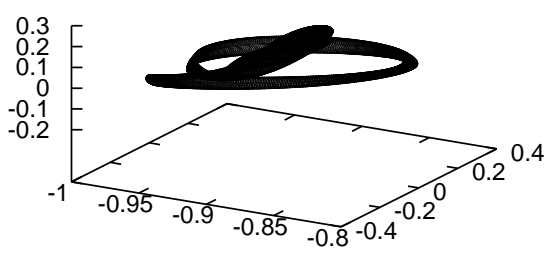

L2, period duplication

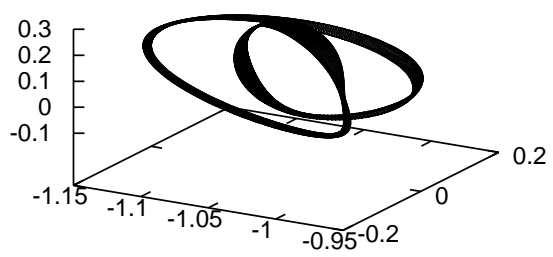

L1, period triplication

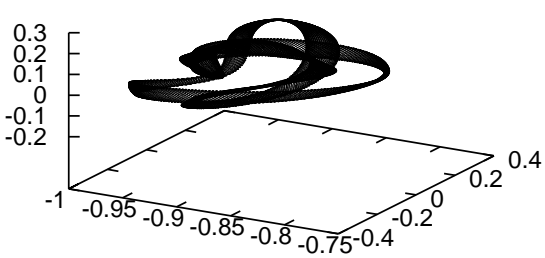

L2, period triplication

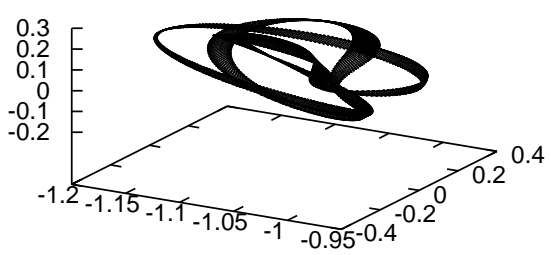

Figure 21: Tori around the bifurcated halo-type orbits. The two on the top are in the families around $L_{1}$ and have energy $h=-1.501$ and rotation numbers $\rho=0.54081$ (left) and $\rho=0.59175$. The two on the bottom are in the families around $L_{2}$ and have energy $h=-1.507$ and rotation numbers $\rho=0.35663$ and $\rho=0.33748$.

\section{Summary of results}

In order to compare the obtained results with the ones computed in previous works using the reduction to the central manifold $([5],[11])$, in this section we will show the evolution (with respect to the energy) of the behavior of the Poincaré sections through $z=0, p_{z}>0$, of all the different sorts of orbits computed.

Figures 22, 23 and 24 show the results for $L_{1}, L_{2}$ and $L_{3}$, respectively. In all these figures we have represented the $x-y$ coordinates at the intersections with $z=0, p_{z}>0$. All the plots have a similar structure. The exterior curve in each plot is the Lyapunov planar orbit of the energy level corresponding to the plot. As this orbit is planar, it is completely included in the surface of section, and is the only orbit for which this happens. The motion inside the region bounded by the Lyapunov planar orbit is quasi-periodic, except at some gaps which cannot be distinguished from the pictures. In all the plots there is a fixed point on the $x$ axis associated to the vertical Lyapunov orbit.

For the three equilibrium points, and for small energy values, the whole picture is formed by invariant curves surrounding the fixed point associated to the vertical orbit. They are associated to the intersections of the Lissajous type trajectories around the vertical periodic orbit, whose 

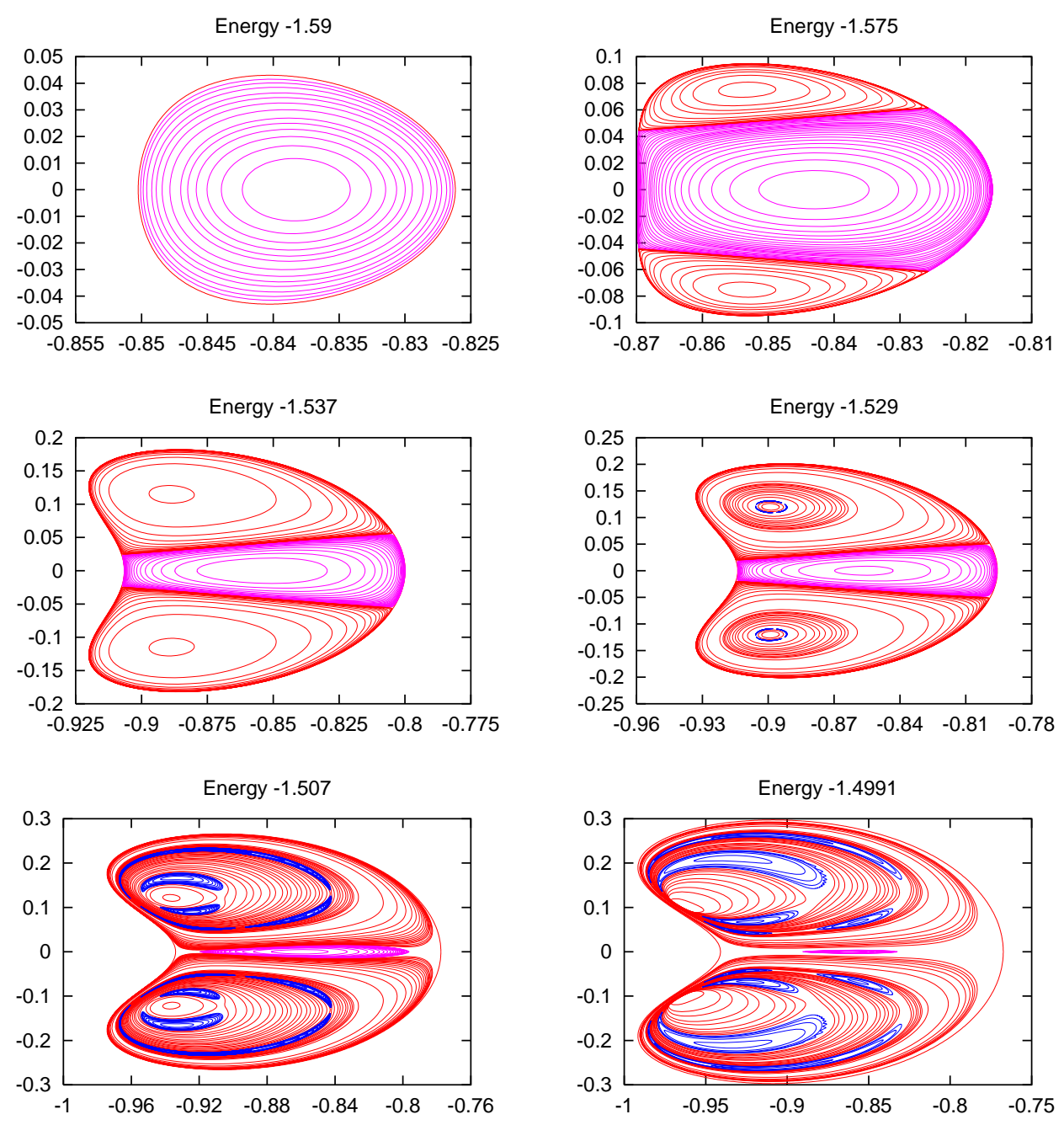

Figure 22: Energy slices of the section $z=0, p_{z}>0$ of the invariant tori around $L_{1}$ computed in the previous section. 

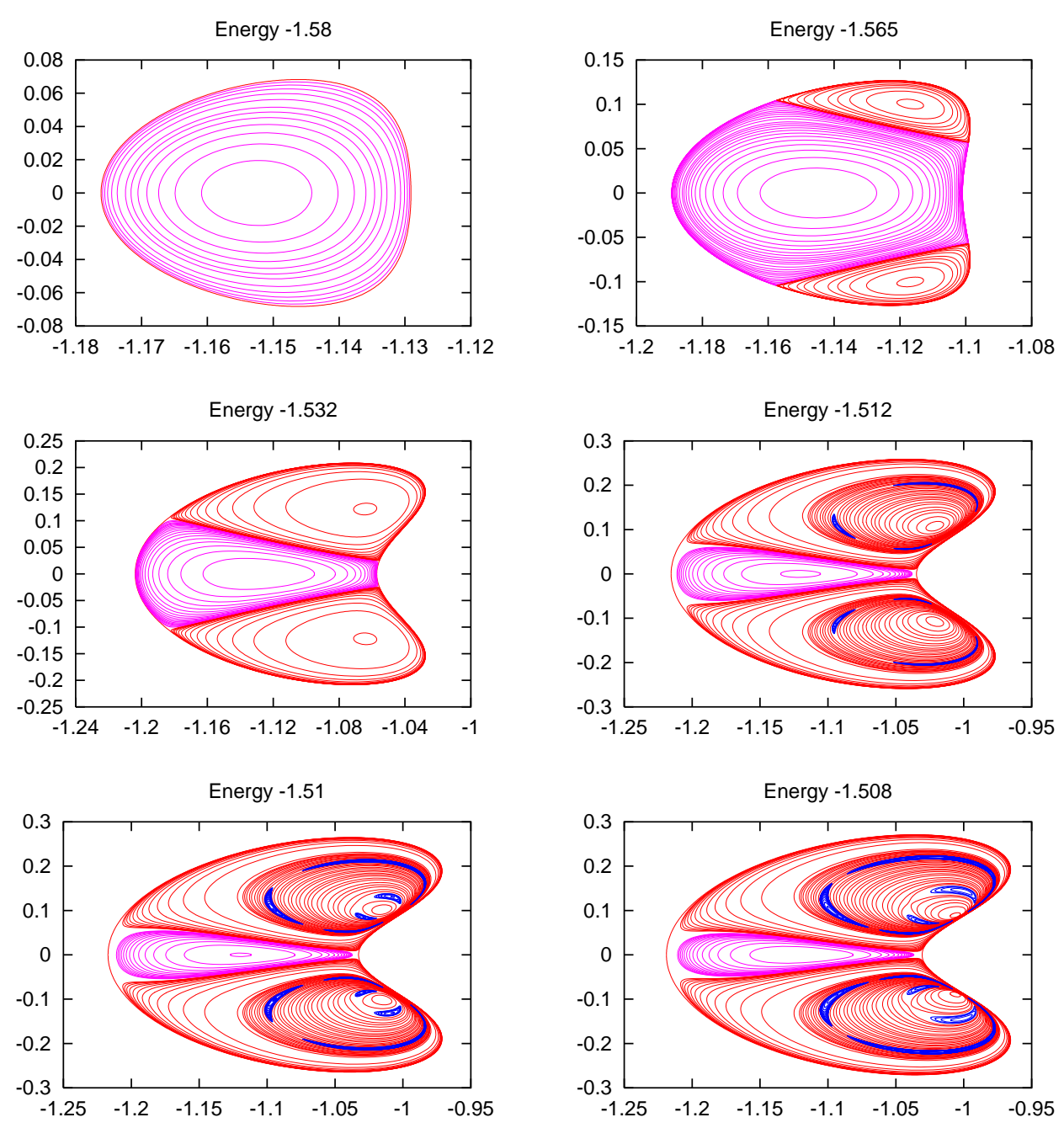

Figure 23: Energy slices of the section $z=0, p_{z}>0$ of the invariant tori around $L_{2}$ computed in the previous section. 

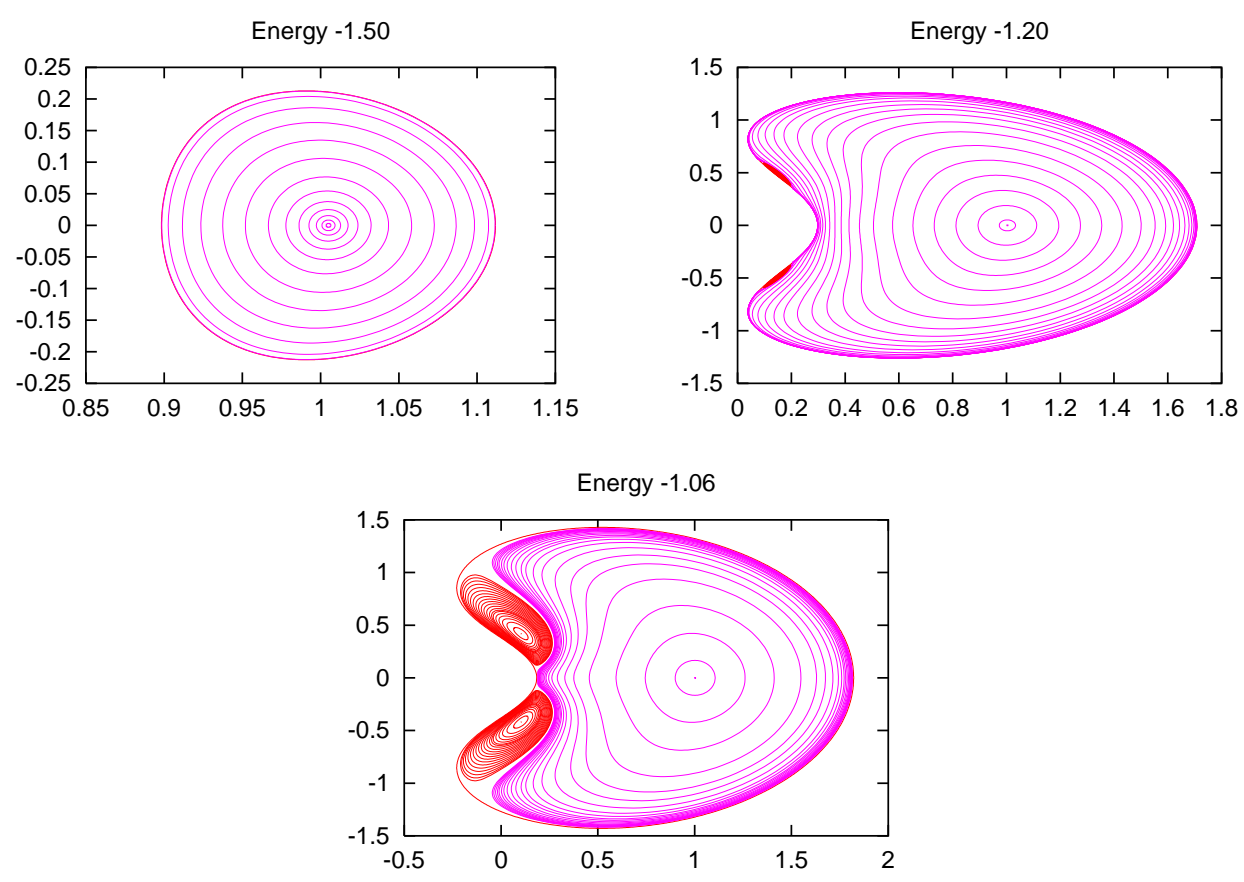

Figure 24: Energy slices of the section $z=0, p_{z}>0$ of the invariant tori around $L_{3}$ computed in the previous section.

evolution from the planar Lyapunov periodic orbits to the vertical one was displayed in figure 16. At the energy levels associated to the first bifurcation of the Lyapunov planar family (first bifurcation of table 3) there appear the halo orbits. This can be seen clearly in the Poincaré map representations, since there appear two additional fixed points surrounded by invariant curves. Increasing the values of the energy, as it was discussed in previous sections, the $L_{1}$ and $L_{2}$ families of halo orbits have two relevant bifurcations, by period triplication and duplication. Both bifurcations can be also detected on the Poincaré representations. This additional structure has not been detected for the $L_{3}$ case. As it was already said, within the bifurcated families there are some with central part, which are surrounded by invariant tori. These tori give rise to the "island chain" structure typical of two-dimensional area-preserving maps. To display more clearly this behavior, in figures 25 and 26 we display a magnification of the bifurcated periodic orbits and its surrounding invariant tori.

The region between the tori around the vertical Lyapunov orbit and the tori around the halo orbits is not empty, as it appears in the above figures, and should contain, at least, the traces, on the surface of section, of the invariant manifolds of the Lyapunov planar orbit. These manifolds act as separatrices between both kinds of motion. The same thing happens between the islands of the bifurcated halo-type orbits and the tori around the halo orbits. In this case, the region between both kinds of tori is filled with the traces of the invariant manifolds of the bifurcated hyperbolic halo-type orbits. In all these boundary regions, the motion should have a chaotic behavior. With our tools we have not been able to compute these separatrices, which can be found in [8] for values of the energy not too far from the ones of the equilibrium.

Aside from these manifolds, there are some families of invariant tori whose intersections with 
L1, energy -1.529

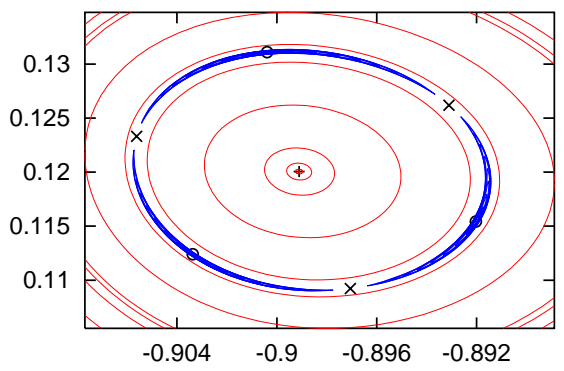

L1, energy -1.507

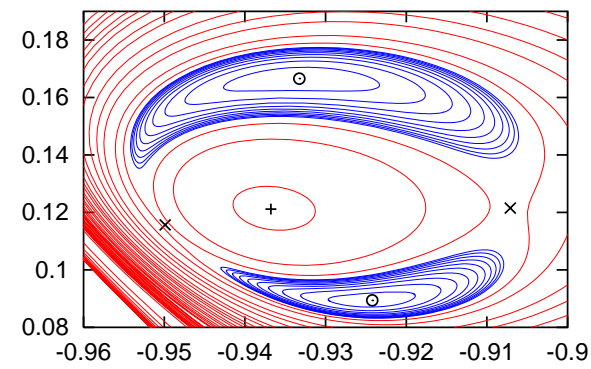

Figure 25: Magnification of two intermediate slices of figure 22 displaying the bifurcated halo-type orbits and the tori surrounding them in the $L_{1}$ case. The points marked with a + represent the $z=0$, $p_{z}>0$ sections of halo orbits, those with $\times$ represent the sections of hyperbolic bifurcations of halo orbits by period duplication or triplication, and the $\odot$ points represent the elliptic ones.
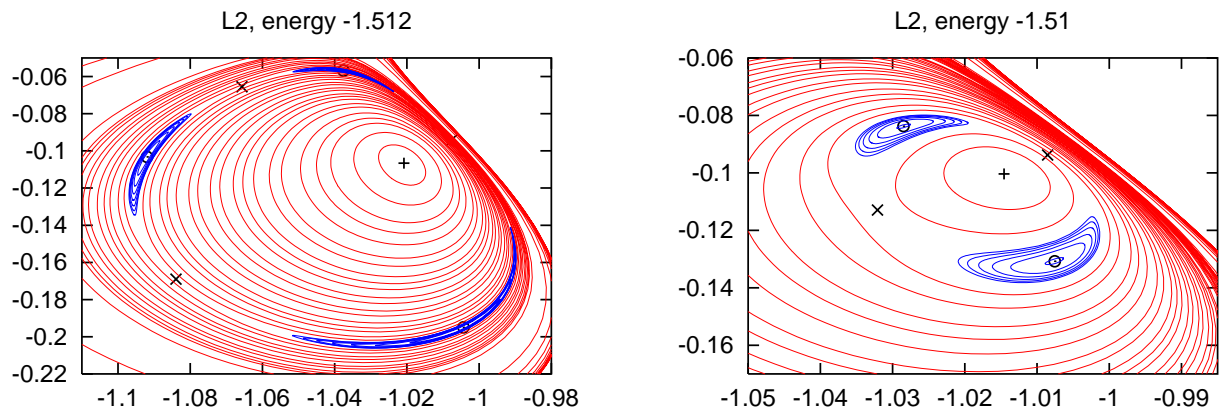

Figure 26: Magnification of two intermediate slices of figure 23 displaying the bifurcated halo-type orbits and the tori surrounding them in the $L_{2}$ case.

$\left\{z=0, \quad p_{z}>0\right\}$ are in these "empty regions". Also, some additional families of tori, whose intersection with the section is outside the outer boundary defined by the planar Lyapunov orbit, will be shown in what follows.

In Fig. 1 we already displayed a more complete picture corresponding to the energy value -1.507 of Fig. 22. For this energy level, the two-lane bridge between the planar and the vertical Lyapunov families of p.o. has already bifurcated, so the planar family has gained central part and, therefore, its periodic orbits are surrounded by invariant tori. The $\{z=0\}$ sections of these tori are the most outer curves that appear in the figure. In it we also see, as two fixed points, the intersections of the two orbits of the bridge with the surface of section. The invariant manifolds of these bicurcated p.o. are the ones that must act as separatrices between the different kinds of tori for this value of the energy.

Following the evolution of the halo family close to the zone displayed in Fig. 10, at the local maximum of the small stability parameter of the halo family there is a turning point in the energy direction, this is for $h=-1.49892$. After this point, the values of the energy decrease up to a second turning point, which happens at $h=-1.50201$. For any value of the energy between -1.50201 and -1.49892 we have three halo orbits, one on the branch before the first turning 
point, another between the two turning points and a third one after the second turning point. We have labeled these branches as 1, 2 and 3 (see Fig. 27). The orbits of branch 1 are surrounded by a two-parametric family of invariant tori, those in branch 2 have two two-parametric families of tori around them (one for each stability parameter) up to the value of the energy -1.49932 , for which the smallest stability parameter reaches the value -2 . After this value, only the family related to the larger stability parameter subsists. The orbits of the third branch have both stability parameters outside $[-2,2]$, therefore there are no invariant tori around them.

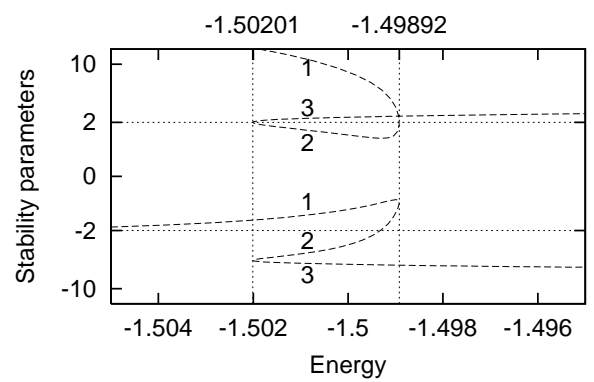

Figure 27: Magnification of the stability parameters of the halo family around $L_{1}$ in the range of energies between the two turning points. We have labeled in each of the stability curves, the branches 1,2 and 3 that are discussed in the text.
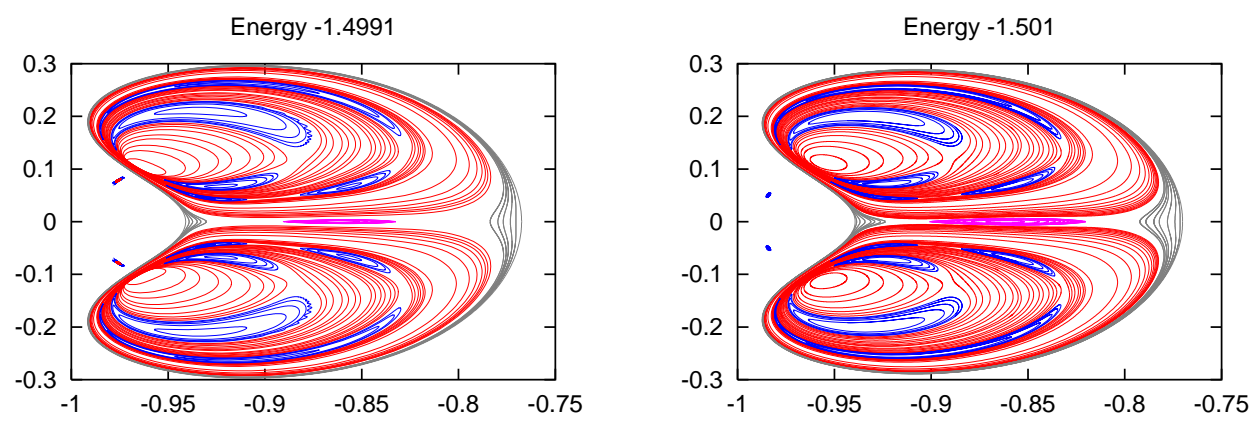

Figure 28: Sections with $\left\{z=0, p_{z}>0\right\}$ of the families of tori around branches 2 and 3 (see text) of the halo family and around the Lyaponov orbits, as well as all the families already displayed in Fig. 22. The sections are done at energy levels -1.4991 and -1.501 .

Fig. 28 shows the intersection with the surface of section of all the above families for the energy levels -1.4991 and -1.501 , which display the two situations above explained. For $h=-1.4991$ the sections of the two families of tori overlap. Their magnifications are given separately in Fig. 29. The computation of both families has been stopped when the number of harmonics required for their determination has exceeded the maximum value allowed (100). Finally, in Fig. 30 we display for the two values of the energy a sample of the torus computed for each family (excluding the tori around the bifurcated halo families obtained by period duplication and triplication). 

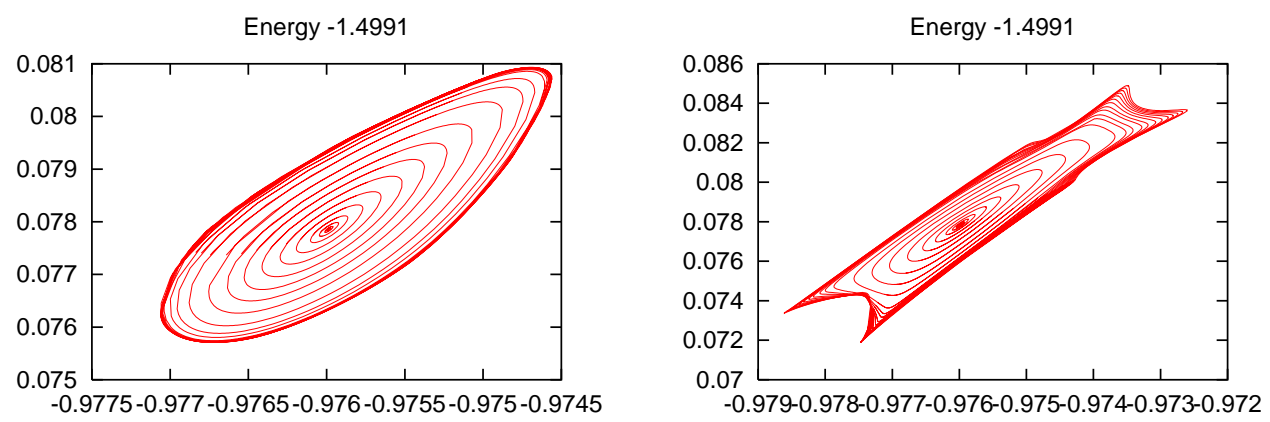

Figure 29: Magnification of the families of tori around branch 2 of the halo family of the left plot of Fig. 28. The left plot corresponds to the smaller stability parameter of the halo family and the right plot to the larger stability parameter

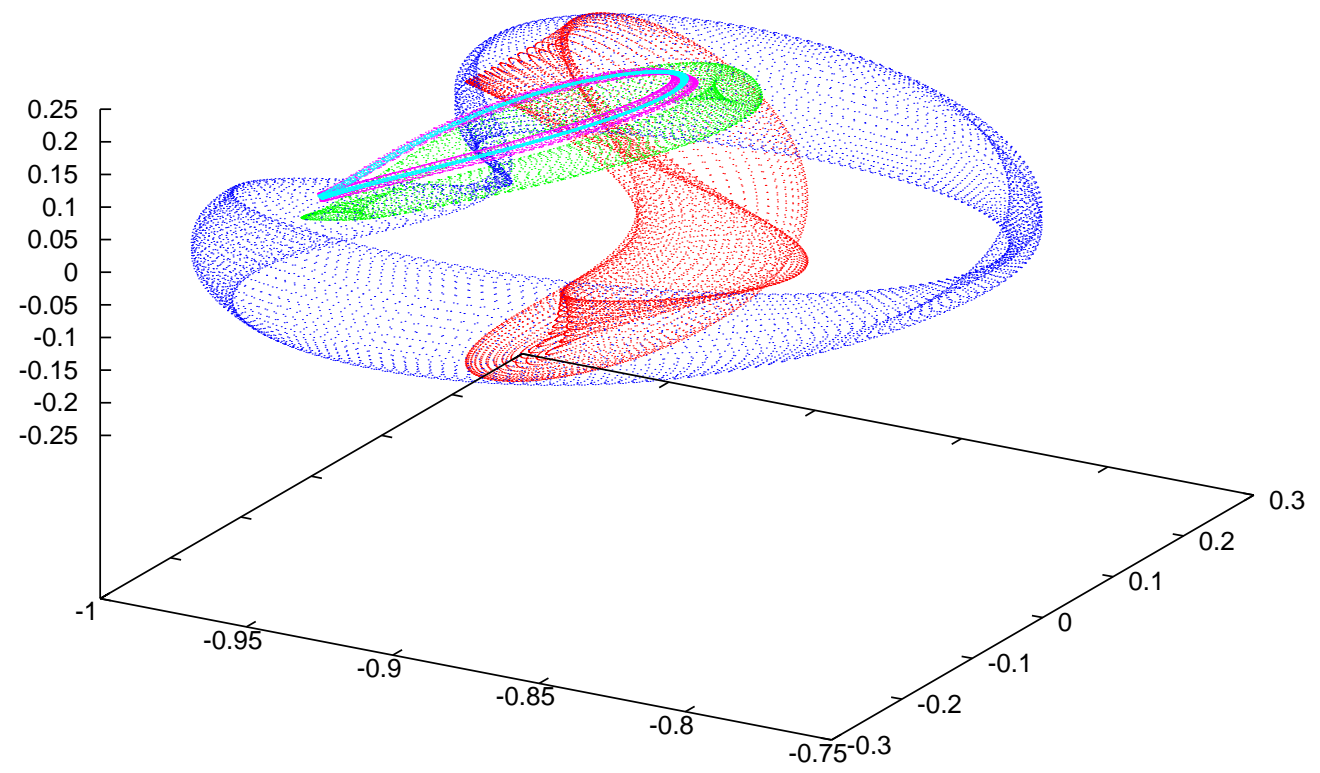

Figure 30: A sample torus of each of the following families: tori around Lyapunov planar orbits, tori around Lyapunov verical orbits, tori around branch 1 of halo orbits (the largest one), tori around branch 2 of halo orbits corresponding to the larger stability parameter (the thickest one of the two above the previous one) and torus around branch 2 of halo orbits corresponding to the smallest stability parameter (the thinest one). 


\section{Acknowledgements}

We are indebted with C. Simó for his careful reading of a first version of this paper and his valuable comments and suggestions. This work has been partially supported by the Catalan grant CIRIT 1996S0GR-00105. Second author's work has been supported by the grant 1997FI 00136 PG from the Generalitat de Catalunya. The numerical simulations have been carried out on Hidra, the Beowulf cluster of the UB/UPC Dynamical Systems Group.

\section{References}

[1] E. Anderson, Z. Bai, C. Bischof, J. Demmel, J. Dongarra, J. D. Croz, A. Greenbaum, S. Hammarling, A. McKenney, S. Ostrouchov, and D. Sorensen. LAPACK Users' Guide. SIAM, second edition, 1995.

[2] L. S. Blackford, J. Choi, A. Cleary, E. D’Azevedo, J. Demmel, I. Dhillon, J. Dongarra, S. Hammarling, G. Henry, A. Petitet, K. Stanley, D. Walker, and R. C. Whaley. ScaLAPACK Users' Guide. SIAM, 1997.

[3] E. Castellà and A. Jorba. On the vertical families of two-dimensional tori near the triangular points of the bicircular problem. Celestial Mechanics and Dynamical Astronomy, 76:35-54, 2000 .

[4] G. H. Golub and C. R. Van Loan. Matrix Computations. The Johns Hopkins University Press, third edition, 1996.

[5] G. Gómez, À. Jorba, J. Masdemont, and C. Simó. Dynamics and Mission Design Near Libration Point Orbits - Volume 3: Advanced Methods for Collinear Points. World Scientific, 2000 .

[6] G. Gómez, A. Jorba, J. Masdemont, and C. Simó. Dynamics and Mission Design Near Libration Point Orbits - Volume 4: Advanced Methods for Triangular Points. World Scientific, 2000 .

[7] G. Gómez, J. Llibre, R. Martínez, and C. Simó. Dynamics and Mission Design Near Libration Point Orbits - Volume 1: Fundamentals: The Case of Collinear Libration Points. World Scientific, 2000.

[8] G. Gómez and J. J. Masdemont. Some zero cost transfers between halo orbits. To appear in Advances in the Astronautical Sciences, 2000.

[9] G. Gómez, J. J. Masdemont, and C. Simó. Quasihalo orbits associated with libration points. Journal of The Astronautical Sciences, 46(2):1-42, 1999.

[10] M. Hénon. Vertical stability of periodic orbits in the restricted problem. Astronomy \& Astrophysics, 28:415-426, 1973.

[11] A. Jorba and J. J. Masdemont. Dynamics in the center manifold of the restricted three-body problem. Physica D, 132:189-213, 1999. 
[12] À. Jorba and J. Villanueva. On the persistence of lower-dimensional invariant tori under quasi-periodic perturbations. Journal of Nonlinear Science, 7:427-473, 1997.

[13] W. S. Koon, M. W. Lo, J. E. Marsden, and S. D. Ross. Heteroclinic connections between periodic orbits and resonance transitions in Celestial Mechanics. Chaos, 10(2):427-469, 2000.

[14] J. Llibre, R. Martínez, and C. Simó. Transversality of the invariant manifolds associated to the Lyapunov family of periodic orbits near $l_{2}$ in the restricted three-body problem. Journal of Differential Equations, 58:104-156, 1985.

[15] D. Richardson. Analytical construction of periodic orbits about the collinear points. Celestial Mechanics, 22(3):241-253, 1980.

[16] C. L. Siegel and J. K. Moser. Lectures on Celestial Mechanics. Springer-Verlag, 1971.

[17] C. Simó. On the analytical and numerical approximation of invariant manifolds. In D. Benest and C. Froeshlé, editors, Modern methods in Celestial Mechanics, pages 285-330. Editions Frontières, 1990.

[18] J. Stoer and R. Bulirsch. Introduction to Numerical Analysis. Springer Verlag, 1983.

[19] V. Szebehely. Theory of orbits. Academic Press, 1967.

[20] C. Zagouras and P. Kazantzis. Three-dimensional periodic oscillations generating from plane periodic ones around the collinear lagrangian points. Astrophysics Space Science, 61(4):389409, 1979. 